\title{
STRONG RATES OF CONVERGENCE FOR A SPACE-TIME DISCRETIZATION OF THE BACKWARD STOCHASTIC HEAT EQUATION, AND OF A LINEAR-QUADRATIC CONTROL PROBLEM FOR THE STOCHASTIC HEAT EQUATION*
}

\author{
ANDREAS PROHL ${ }^{1}$ AND YANQING WANG ${ }^{2, * *}$
}

\begin{abstract}
We verify strong rates of convergence for a time-implicit, finite-element based space-time discretization of the backward stochastic heat equation, and the forward-backward stochastic heat equation from stochastic optimal control. The fully discrete version of the forward-backward stochastic heat equation is then used within a gradient descent algorithm to approximately solve the linearquadratic control problem for the stochastic heat equation driven by additive noise. This work is thus giving a theoretical foundation for the computational findings in Dunst and Prohl, SIAM J. Sci. Comput. 38 (2016) A2725-A2755.
\end{abstract}

Mathematics Subject Classification. 49J20, 65M60, 93E20.

Received September 29, 2020. Accepted May 10, 2021.

\section{INTRODUCTION}

Let $D \subset \mathbb{R}^{d}$ be a bounded domain with $C^{2}$ boundary, $T>0$, and a (deterministic) function $\widetilde{X} \equiv\{\widetilde{X}(t) ; t \in$ $[0, T]\} \in C\left([0, T] ; \mathbb{H}_{0}^{1} \cap \mathbb{H}^{2}\right)$ be given. Our goal is to numerically approximate the $\mathbb{L}^{2}$-valued, $\mathbb{F}$-adapted control process $U^{*} \equiv\left\{U^{*}(t) ; t \in[0, T]\right\}$ on the filtered probability space $(\Omega, \mathcal{F}, \mathbb{F}, \mathbb{P})$ that minimizes the functional $(\alpha \geq 0)$

$$
\mathcal{J}(X, U)=\frac{1}{2} \mathbb{E}\left[\int_{0}^{T}\left(\|X(t)-\widetilde{X}(t)\|_{\mathbb{L}^{2}}^{2}+\|U(t)\|_{\mathbb{L}^{2}}^{2}\right) \mathrm{d} t+\alpha\|X(T)-\widetilde{X}(T)\|_{\mathbb{L}^{2}}^{2}\right]
$$

subject to the (controlled forward) stochastic heat equation (SPDE, for short)

$$
\left\{\begin{array}{l}
\mathrm{d} X(t)=[\Delta X(t)+U(t)] \mathrm{d} t+\sigma(t) \mathrm{d} W(t) \quad \forall t \in[0, T] \\
X(0)=X_{0}
\end{array}\right.
$$

* This work is supported in part by the National Natural Science Foundation of China $(11801467,11701470)$, and the Chongqing Natural Science Foundation (cstc2018jcyjAX0148).

Keywords and phrases: Strong error estimate with rates, backward stochastic heat equation, stochastic linear quadratic problem, forward-backward stochastic heat equation.

1 Mathematisches Institut, Universität Tübingen, Auf der Morgenstelle 10, 72076 Tübingen, Germany.

2 School of Mathematics and Statistics, Southwest University, Chongqing 400715, PR China.

** Corresponding author: yqwang@amss.ac.cn 
which is supplemented by homogeneous Dirichlet boundary conditions. Here $W \equiv\{W(t) ; t \in[0, T]\}$ is an $\mathbb{R}^{m}$-valued Wiener process, and $X_{0} \in \mathbb{H}_{0}^{1} \cap \mathbb{H}^{2}$, and let $\sigma \equiv\left\{\left(\sigma_{1}(t), \sigma_{2}(t), \ldots, \sigma_{m}(t)\right) ; t \in[0, T]\right\}$, with $\sigma_{i} \in$ $L_{\mathbb{F}}^{\infty}\left(0, T ; L^{2}\left(\Omega ; \mathbb{H}_{0}^{1} \cap \mathbb{H}^{2}\right)\right)$ for $i=1,2, \ldots, m$. For every $U \in L_{\mathbb{F}}^{2}\left(\Omega ; L^{2}\left(0, T ; \mathbb{L}^{2}\right)\right)$, there exists a unique $\mathbb{H}_{0}^{1}$ valued mild/weak solution $X \equiv X(U) \in L_{\mathbb{F}}^{2}\left(\Omega ; C\left([0, T] ; \mathbb{L}^{2}\right) \cap L^{2}\left(0, T ; \mathbb{H}_{0}^{1}\right)\right)$ in (1.2) (see e.g. [26]), and also a unique minimizer $\left(X^{*}, U^{*}\right) \in L_{\mathbb{F}}^{2}\left(\Omega ; C\left([0, T] ; \mathbb{L}^{2}\right) \cap L^{2}\left(0, T ; \mathbb{H}_{0}^{1}\right) \times L^{2}\left(0, T ; \mathbb{L}^{2}\right)\right)$ of the stochastic optimal control problem: 'minimize (1.1) subject to (1.2)' can be deduced-which we below refer to as SLQ (see e.g. [5]).

We consider problem SLQ as a prototype example of a (linear-quadratic) stochastic optimal control problem involving a stochastic PDE, for which corresponding numerical analyses so far are rare in the existing literature; see e.g. $[15,35]$. This is in contrast to the deterministic counterpart problem $\mathbf{L Q}$ which involves a linear PDE, where optimal rates of convergence are available for (finite-element based) space-time discretization of related optimality conditions (see e.g. [20, 27-29,32]), which may then be used as part of a gradient descent algorithm with step size control [21] to approximate the minimizing tuple $\left(X^{*}, U^{*}\right)$, which here consists of deterministic state and control functions. If compared to problem LQ, problem SLQ owns some distinctive characters and additional difficulties caused by the driving Wiener process in the SPDE (1.2), which make the generalization of the numerical results for the deterministic control problem to SLQ a non-trivial task. For example, a crucial difficulty consists in solving the adjoint equation in the context of SLQ, which here is a backward stochastic PDE (BSPDE, for short) of the form

$$
\left\{\begin{array}{l}
\mathrm{d} Y(t)=[-\Delta Y(t)+[X(t)-\widetilde{X}(t)]] \mathrm{d} t+Z(t) \mathrm{d} W(t) \quad \forall t \in[0, T] \\
Y(T)=-\alpha(X(T)-\widetilde{X}(T)),
\end{array}\right.
$$

having a unique solution tuple $(Y, Z) \in L_{\mathbb{F}}^{2}\left(\Omega ; C\left([0, T] ; \mathbb{H}_{0}^{1}\right) \cap L^{2}\left(0, T ; \mathbb{H}_{0}^{1} \cap \mathbb{H}^{2}\right)\right) \times L_{\mathbb{F}}^{2}\left(\Omega ; L^{2}\left(0, T ; \mathbb{H}_{0}^{1}\right)\right) ; c f$. [14]. The adjoint variable $Y$ is then related to the optimal control by Pontryagin's maximum principle, which in the case of problem SLQ is

$$
0=U^{*}(t)-Y(t) \quad \forall t \in(0, T) .
$$

The combination of equations (1.2), (1.3), and (1.4) then uniquely determines the optimal process tuple $\left(X^{*}, U^{*}\right)$ of problem SLQ.

Both, the numerical discretization and related analysis of SLQ governed by SPDEs are non-trivial. In this work, we apply Pontryagin's maximum principle to discretize problem SLQ. There exists related works to approximate optimal control problems driven by SDE systems; see, e.g., [1, 2, 19]; however, to the best of the authors' knowledge, works on discretization of SLQ with SPDEs constraints so-far are rare. In [15], a scheme to numerically solve (a slightly different) problem is given, which combines a proper selection of involved finite element spaces with an implicit Euler method for temporal discretization to inherit relevant stability properties of the limiting system, with a least squares Monte-Carlo method, in combination with a stochastic gradient method, which is based on the (discretization of) optimality system (1.2), (1.3), and (1.4). The scheme was implemented in [15] for a problem that uses a two-dimensional bounded domain $D$, and comprehensive computational studies are reported, among which evidence is given for

a) strong convergence order $\frac{1}{2}$ for the iterates of both, the temporal discretization of BSPDE, and SLQ (with the parameter $\tau>0$ ),

b) optimal strong convergence order for the iterates of both, the spatial discretization of BSPDE, and SLQ (with the parameter $h>0$ ), depending on the spatial $\mathbb{L}^{2}$ - or $\mathbb{H}^{1}$-norm, in which the error is considered.

By evidence, a convergence rate as stated in a) may be expected from the literature on backward stochastic (ordinary) differential equations (BSDEs, for short), where the dimension of the involved state spaces is fixed; see e.g. [39], and the discussion below. In the present setting of BSPDE and SLQ, however, where solutions take values in (infinite dimensional) Hilbert spaces, the related dimensions in a (finite-element based) discretization depend on the inverse of the discretization parameter $h>0$, and a naive transfer of results from [39] would 
result in error estimates which couple temporal and spatial discretization parameters - which severely restricts the wanted flexibility of spatio-temporal meshes to resolve relevant multiscale solution features. In this work, we use Malliavin calculus and variational analysis as technical tools to exploit inherent stability properties of timeimplicit finite-element based discretizations to validate strong error estimates for (the space-time discretization of) both problems, where both discretization parameters enter additively, and no coupling terms arise. As such, this work is meant to give a first theoretical guidance on the design and analysis of numerical methods for problems BSPDE and SLQ, as well as an explanation for the computational findings in [15] — where no couplings of discretization parameters $(\tau, h)$ were observed, thus allowing for general quasiuniform triangulations of $D$ for example, in the computational studies for (slightly different version of) BSPDE and SLQ; see also Section 4 below.

Conceptually, we start our work below with the numerical analysis of BSPDE, which is the crucial building block for the (numerical analysis of the) optimality conditions (1.2), (1.3), and (1.4) for SLQ, which is then considered afterwards. There is a rich body of literature on numerical schemes for BSDEs; see e.g. [3, 6, $9,11,16,18,22,33,37,39,41]$, and the references therein. In contrast, the convergence analysis of spacetime discretizations of BSPDE is only a recent research subject, and available results are rare: we are only aware of the works [34, 35], where a first error analysis for an (abstract) time-space discretization based on the implicit Euler method for the above BSPDE (1.3) is proposed, where the error depends on the ratio of temporal discretization and Galerkin parameters; see our discussion above. In [15], the authors derive rates of convergence for a conforming finite element semi-discretization, and discuss its actual implementation. The proofs in [15] use simple variational arguments, resting on improved regularity properties of the variational solution, Itô's formula, and approximation results for the finite element method. However, the interplay of spatial and temporal discretization errors was left open in [15], in particular the relevant question regarding unconditional convergence rates (which were discussed above), which allow discretization parameters w.r.t. time and space to independently tend to zero, and general quasiuniform space-time meshes.

A stability and strong error analysis for the spatial semi-discretization (2.14) already exists in the literature ( $c f$. Sect. 2.3 of a summary); however, the role of an additional temporal discretization of (2.14) was not theoretically accessible in [15]; see again our discussion above. In Section 3, we fill this gap by using Malliavin calculus for the (finite-element type) solutions $\left(Y_{h}, Z_{h}\right)$ of (2.14) to derive estimates for its increments in relevant norms, such as the uniform estimate (see Lem. 3.2)

$$
\sup _{h>0} \mathbb{E}\left[\left\|Z_{h}(t)-Z_{h}(s)\right\|_{\mathbb{L}^{2}}^{2}\right] \leq C|t-s|
$$

One key tool to validate this estimate for the solution $\left(Y_{h}, Z_{h}\right)$ of (2.14) is the observation that $Z_{h}$ is the Malliavin derivative of $Y_{h}$, and the Malliavin derivative of $\left(Y_{h}, Z_{h}\right)$ also satisfies a BSPDE (see Lem. 2.2); another tool is the auxiliary equation (3.7), which allows to subsequently study of temporal discretization effects on the first, and the second solution component of the solutions $\left(Y_{h}, Z_{h}\right)$ of $(2.14)$. We use this again in Section 4 for the study the space-time discretization effects for FBSPDE; $c f$. (4.32).

The second goal in this work is addressed in Section 4, where strong error estimates for a space-time discretization (4.15)-(4.16) of the coupled forward-backward SPDE (1.2)-(1.3) (FBSPDE for short) are shown: its derivation starts ( $c f$. Sect. 4.1) with the error analysis of a spatial semi-discretization (4.5)-(4.7) of the optimality system FBSPDE of optimization problem SLQ, where our main result are optimal error estimates in Theorem 4.1. Conceptionally, its proof first deduces estimates for the (discrete) optimal control (see (i) in Thm. 4.1) via the use of the (strictly convex, differentiable) reduced functional $\widehat{\mathcal{J}}_{h}$ in (4.8), in particular, the representation of its derivative in (4.9), and stability properties (uniformly in $h$ ) of the spatial discretizations of the forward, and the adjoint/backward problem (see Sect. 3) in the given context of finite elements. Once (i) of Theorem 4.1 is settled, the estimates (ii) and (iii) now essentially follow from Section 3. Section 4.2 then focuses on the error due to (additional) temporal discretization of (4.5)-(4.7) in the form (4.15)-(4.16), which-as for the BSPDE case -, is much more intricate: the key tools to validate the main result here (i.e., Thm. 4.3) are a representation of the derivative of the reduced functional $\widehat{\mathcal{J}}_{h \tau}$ in (4.26) via (4.15), together with stability and 
convergence estimates from Section 3 to show (i) in Theorem 4.3. Statements (ii) and (iii) in Theorem 4.3 then follow from the results in Section 3 by separately considering the (space-time) discretization of the forward, and the adjoint/backward equation, and using Theorem 3.5, in particular. The results of Section 4 therefore establish optimal rates of convergence for the minimizer of the space-time discrete optimization problem SLQ $\mathbf{Q}_{h \tau}$ (see (4.13)-(4.14)) towards the minimizer of SLQ.

We remark that even for numerical methods of coupled forward-backward stochastic differential (ordinary) equations (SDEs, for short), the convergence analysis is non-trivial, and to obtain rates of convergence, usually assumptions such as small time intervals $[0, T]$ are needed (see e.g. [4]). In the present setting, the results in Section 4 extend available ones (cf. [15]) in the literature in several aspects: the obtained strong convergence rates for the used finite element based space-time discretization (4.15) hold for arbitrary times $T$-and is not only a semi-discretization in space where optimal rates are obtained in [15] for small times $T$ via a contraction argument.

To solve a BSPDE computationally requires huge computational resources (see [15]), and it is even more computationally demanding (in terms of computational storage requirements and computational times) to solve the coupled FBSPDE. Consequently, an alternative numerical strategy to the space-time discretization (4.15)(4.16) of FBSPDE which couples the forward and the backward part is needed in practice. While a simple fixed-point method on the level of optimality conditions to accomplish this goal is known to converge only for small times $T>0(c f .[4,15])$, the construction of a decoupled system should be based on the fully discretized problem $\mathbf{S L Q}_{h \tau}$ (4.13)-(4.14), where convergence of a gradient descent method exploits its character as a minimization problem, and allows for computational decouplings; we refer to Section 5 for details of this iterative scheme, and its convergence, which is the final goal in this work.

The rest of this paper is organized as follows. In Section 2, we introduce notations, and review relevant properties of the problems BSPDE (2.10) and FBSPDE considered in this work. In Section 3, we prove strong error estimates for a space-time discretization of BSPDE. By virtue of the obtained error estimates, in Section 4, we prove a convergence rate for a space-time discretization of FBSPDE, which is related to problem SLQ. Convergence of the related iterative gradient descent method towards the minimizer $U^{*}$ of SLQ is shown in Section 5.

\section{PRELiminaries}

\subsection{Notation - involved processes and the finite element method}

Let $\left(\mathbb{K},(\cdot, \cdot)_{\mathbb{K}}\right)$ be a separable Hilbert space. By $\|\cdot\|_{\mathbb{L}^{2}}$ resp. $(\cdot, \cdot)_{\mathbb{L}^{2}}$, we denote the norm resp. the scalar product in Lebesgue space $\mathbb{L}^{2}:=L^{2}(D)$. The norm in sobolev space $\mathbb{H}_{0}^{1}:=H_{0}^{1}(D), \mathbb{H}^{2}:=H^{2}(D)$ is denoted by $\|\cdot\|_{\mathbb{H}_{0}^{1}}$, $\|\cdot\|_{\mathbb{H}^{2}}$ respectively. Let $(\Omega, \mathcal{F}, \mathbb{F}, \mathbb{P})$ be a complete filtered probability space, where $\mathbb{F}=\left\{\mathcal{F}_{t}\right\}_{t \in[0, T]}$ is the filtration generated by the $\mathbb{R}^{m}$-valued Wiener process $W$, which is augmented by all the $\mathbb{P}$-null sets. Below, we set $m=1$ for simplicity. The space of all $\mathbb{F}$-adapted processes $X: \Omega \times[0, T] \rightarrow \mathbb{K}$ satisfying $\mathbb{E}\left[\int_{0}^{T}\|X(t)\|_{\mathbb{K}}^{2} \mathrm{~d} t\right]<\infty$ is denoted by $L_{\mathbb{F}}^{2}\left(\Omega ; L^{2}(0, T ; \mathbb{K})\right)$; the space of all $\mathbb{F}$-adapted processes $X: \Omega \times[0, T] \rightarrow \mathbb{K}$ satisfying $\mathbb{E}\left[\sup _{t \in[0, T]}\|X(t)\|_{\mathbb{K}}^{2}\right]<$ $\infty$ is denoted by $L_{\mathbb{F}}^{2}(\Omega ; C([0, T] ; \mathbb{K}))$.

We partition the bounded domain $D \subset \mathbb{R}^{d}$ via a regular triangulation $\mathcal{T}_{h}$ into elements $K$ with maximum mesh size $h:=\max \left\{\operatorname{diam}(K): K \in \mathcal{T}_{h}\right\}$, and consider spaces

$$
\mathbb{V}_{h}^{1}:=\left\{\phi \in \mathbb{H}_{0}^{1}:\left.\phi\right|_{K} \in \mathbb{P}_{1}(K) \quad \forall K \in \mathcal{T}_{h}\right\}, \quad \mathbb{V}_{h}^{0}:=\left\{\phi \in \mathbb{L}^{2}:\left.\phi\right|_{K} \in \mathbb{P}_{0}(K) \quad \forall K \in \mathcal{T}_{h}\right\},
$$

where $\mathbb{P}_{i}(K)$ denotes the space of polynomials of degree $i(i=0,1)$. The $\mathbb{L}^{2}$-projection $\Pi_{h}^{i}: \mathbb{L}^{2} \rightarrow \mathbb{V}_{h}^{i}$ is defined by $\left(\Pi_{h}^{i} \xi-\xi, \phi_{h}\right)_{\mathbb{L}^{2}}=0$ for all $\phi_{h} \in \mathbb{V}_{h}^{i}$. We define the discrete Laplacean $\Delta_{h}: \mathbb{V}_{h}^{1} \rightarrow \mathbb{V}_{h}^{1}$ by $\left(-\Delta_{h} \xi_{h}, \phi_{h}\right)_{\mathbb{L}^{2}}=$ $\left(\nabla \xi_{h}, \nabla \phi_{h}\right)_{\mathbb{L}^{2}}$ for all $\xi_{h}, \phi_{h} \in \mathbb{V}_{h}^{1}$.

We use approximation estimates for the projection $\Pi_{h}^{1}$, and an inverse estimate $(c f .[8])$ to conclude that

$$
\left\|\Delta_{h} \Pi_{h}^{1} \xi\right\|_{\mathbb{L}^{2}} \leq C\left\|\nabla^{2} \xi\right\|_{\mathbb{L}^{2}} \quad \forall \xi \in \mathbb{H}_{0}^{1} \cap \mathbb{H}^{2},
$$


since

$$
\begin{aligned}
\left\|\Delta_{h} \Pi_{h}^{1} \xi\right\|_{\mathbb{L}^{2}}^{2} & =-\left(\nabla\left[\Pi_{h}^{1} \xi-\xi\right], \nabla \Delta_{h} \Pi_{h}^{1} \xi\right)_{\mathbb{L}^{2}}-\left(\nabla \xi, \nabla \Delta_{h} \Pi_{h}^{1} \xi\right)_{\mathbb{L}^{2}} \\
& \leq C h\left\|\nabla^{2} \xi\right\|_{\mathbb{L}^{2}}\left\|\nabla \Delta_{h} \Pi_{h}^{1} \xi\right\|_{\mathbb{L}^{2}}+\left(\Delta \xi, \Delta_{h} \Pi_{h}^{1} \xi\right)_{\mathbb{L}^{2}} \\
& \leq C\left(\left\|\nabla^{2} \xi\right\|_{\mathbb{L}^{2}}+\|\Delta \xi\|_{\mathbb{L}^{2}}\right)\left\|\Delta_{h} \Pi_{h}^{1} \xi\right\|_{\mathbb{L}^{2}} .
\end{aligned}
$$

We denote by $I_{\tau}=\left\{t_{n}\right\}_{n=0}^{N} \subset[0, T]$ a time mesh with maximum step size $\tau:=\max \left\{t_{n+1}-t_{n}: n=\right.$ $0,1, \ldots, N-1\}$, and $\Delta_{n} W=W\left(t_{n}\right)-W\left(t_{n-1}\right)$ for all $n=1, \ldots, N$. For simplicity, we choose a uniform partition, i.e. $\tau=T / N$ and $\tau \leq 1$. The results in this work still hold for general partitions.

\subsection{The stochastic heat equation - strong convergence rates for a space-time discretization}

To obtain a convergence rate for the space-time discretization of (1.2), we assume that $X_{0} \in \mathbb{H}_{0}^{1} \cap \mathbb{H}^{2}$, that $U \in L_{\mathbb{F}}^{2}\left(\Omega ; L^{2}\left(0, T ; \mathbb{H}_{0}^{1}\right)\right)$, and $\sigma \in L_{\mathbb{F}}^{\infty}\left(0, T ; L^{2}\left(\Omega ; \mathbb{H}_{0}^{1} \cap \mathbb{H}^{2}\right)\right)$. Under the above assumptions, SPDE (1.2) admits a unique strong solution $X \in L_{\mathbb{F}}^{2}\left(\Omega ; C\left([0, T] ; \mathbb{H}_{0}^{1}\right) \cap L^{2}\left(0, T ; \mathbb{H}_{0}^{1} \cap \mathbb{H}^{2}\right)\right)$, see e.g. [13, 26, 40], such that

$$
\mathbb{E}\left[\sup _{t \in[0, T]}\|X(t)\|_{\mathbb{H}_{0}^{1}}^{2}+\int_{0}^{T}\|X(t)\|_{\mathbb{H}^{2}}^{2} \mathrm{~d} t\right] \leq C \mathbb{E}\left[\left\|X_{0}\right\|_{\mathbb{H}_{0}^{1}}^{2}+\int_{0}^{T}\|U(t)\|_{\mathbb{L}^{2}}^{2} \mathrm{~d} t\right]
$$

where $C \equiv C(D, T)>0$, which satisfies the following weak form $\mathbb{P}$-a.s. for all $t \in[0, T]$

$$
\begin{gathered}
(X(t), \phi)_{\mathbb{L}^{2}}-\left(X_{0}, \phi\right)_{\mathbb{L}^{2}}+\int_{0}^{t}\left[(\nabla X(s), \nabla \phi)_{\mathbb{L}^{2}}-(U(s), \phi)_{\mathbb{L}^{2}}\right] \mathrm{d} s \\
=\int_{0}^{t}(\sigma(s), \phi)_{\mathbb{L}^{2}} \mathrm{~d} W(s) \quad \forall \phi \in \mathbb{H}_{0}^{1} .
\end{gathered}
$$

A finite element discretization of (2.3) then reads: For all $t \in[0, T]$, find $X_{h} \in L_{\mathbb{F}}^{2}\left(\Omega ; C\left([0, T] ; \mathbb{V}_{h}^{1}\right)\right)$ such that $\mathbb{P}$-a.s. and for all times $t \in[0, T]$

$$
\begin{gathered}
\left(X_{h}(t), \phi_{h}\right)_{\mathbb{L}^{2}}-\left(X_{0}, \phi_{h}\right)_{\mathbb{L}^{2}}+\int_{0}^{t}\left[\left(\nabla X_{h}(s), \nabla \phi_{h}\right)_{\mathbb{L}^{2}}-\left(U(s), \phi_{h}\right)_{\mathbb{L}^{2}}\right] \mathrm{d} s \\
=\int_{0}^{t}\left(\sigma(s), \phi_{h}\right)_{\mathbb{L}^{2}} \mathrm{~d} W(s) \quad \forall \phi_{h} \in \mathbb{V}_{h}^{1} .
\end{gathered}
$$

Equation (2.4) may be recast into the following SDE system,

$$
\left\{\begin{array}{l}
d X_{h}(t)=\left[\Delta_{h} X_{h}(t)+\Pi_{h}^{1} U(t)\right] \mathrm{d} t+\Pi_{h}^{1} \sigma(t) \mathrm{d} W(t) \quad \forall t \in[0, T], \\
X_{h}(0)=\Pi_{h}^{1} X_{0}
\end{array}\right.
$$

Thanks to this equivalence, we do not distinguish between $\mathbf{S P D E}_{h}(2.4)$ and SDE (2.5) throughout this paper.

The derivation of a strong error estimate is standard, and uses the improved (spatial) regularity properties of the strong variational solution,

$$
\sup _{t \in[0, T]} \mathbb{E}\left[\left\|X_{h}(t)-X(t)\right\|_{\mathbb{L}^{2}}^{2}\right]+\mathbb{E}\left[\int_{0}^{T}\left\|\nabla\left[X_{h}(t)-X(t)\right]\right\|_{\mathbb{L}^{2}}^{2} \mathrm{~d} t\right] \leq C h^{2}
$$


We now consider a time-implicit discretization of (2.4) on a partition $I_{\tau}$ of $[0, T]$. The problem then reads: For every $0 \leq n \leq N-1$, find a solution $X_{h}^{n+1} \in L_{\mathcal{F}_{t_{n+1}}}^{2}\left(\Omega ; \mathbb{V}_{h}^{1}\right)$ such that $\mathbb{P}$-a.s.

$$
\left(X_{h}^{n+1}-X_{h}^{n}, \phi_{h}\right)_{\mathbb{L}^{2}}+\tau\left[\left(\nabla X_{h}^{n+1}, \nabla \phi_{h}\right)_{\mathbb{L}^{2}}-\left(U\left(t_{n}\right), \phi_{h}\right)_{\mathbb{L}^{2}}\right]=\left(\sigma\left(t_{n}\right), \phi_{h}\right)_{\mathbb{L}^{2}} \Delta_{n+1} W,
$$

where $\Delta_{n+1} W:=W\left(t_{n+1}\right)-W\left(t_{n}\right)$. The verification of the error estimate (see [36])

$$
\max _{0 \leq n \leq N} \mathbb{E}\left[\left\|X_{h}\left(t_{n}\right)-X_{h}^{n}\right\|_{\mathbb{L}^{2}}^{2}\right]+\tau \sum_{n=1}^{N} \mathbb{E}\left[\left\|\nabla\left[X_{h}\left(t_{n}\right)-X_{h}^{n}\right]\right\|_{\mathbb{L}^{2}}^{2}\right] \leq C \tau
$$

rests on stability properties of the implicit Euler, as well as the bound

$$
\sum_{n=0}^{N-1} \int_{t_{n}}^{t_{n+1}} \mathbb{E}\left[\left\|X_{h}(t)-X_{h}\left(t_{n}\right)\right\|_{\mathbb{H}_{0}^{1}}^{2}\right] \mathrm{d} t \leq C \tau,
$$

which requires additional regularity properties of involved data, i.e.,

$$
\sum_{n=0}^{N-1} \int_{t_{n}}^{t_{n+1}} \mathbb{E}\left[\left\|U(t)-U\left(t_{n}\right)\right\|_{\mathbb{L}^{2}}^{2}+\left\|\sigma(t)-\sigma\left(t_{n}\right)\right\|_{\mathbb{L}^{2}}^{2}\right] \mathrm{d} t \leq C \tau,
$$

and the $\mathbb{H}^{1}$-stability of the $\mathbb{L}^{2}$-projection $\Pi_{h}^{1} ; c f .[7,10]$.

\subsection{The backward stochastic heat equation — a finite element based spatial discretization}

Let $Y_{T} \in L_{\mathcal{F}_{T}}^{2}\left(\Omega ; \mathbb{H}_{0}^{1}\right)$ and $f \in L_{\mathbb{F}}^{2}\left(\Omega ; L^{2}\left(0, T ; \mathbb{L}^{2}\right)\right)$. A strong solution to the backward stochastic heat equation

$$
\left\{\begin{array}{l}
\mathrm{d} Y(t)=[-\Delta Y(t)+f(t)] \mathrm{d} t+Z(t) \mathrm{d} W(t) \quad \forall t \in[0, T] \\
Y(T)=Y_{T}
\end{array}\right.
$$

is a pair of square integrable $\mathbb{F}$-adapted processes $(Y, Z) \in L_{\mathbb{F}}^{2}\left(\Omega ; C\left([0, T] ; \mathbb{H}_{0}^{1}\right) \cap L_{\mathbb{F}}^{2}\left(0, T ; \mathbb{H}_{0}^{1} \cap \mathbb{H}^{2}\right)\right) \times$ $L_{\mathbb{F}}^{2}\left(0, T ; \mathbb{H}_{0}^{1}\right)$, and there exists a constant $C \equiv C(D, T)>0$ such that

$$
\mathbb{E}\left[\sup _{t \in[0, T]}\|Y(t)\|_{\mathbb{H}_{0}^{1}}^{2}\right]+\mathbb{E}\left[\int_{0}^{T}\|Y(t)\|_{\mathbb{H}^{2}}^{2}+\|Z(t)\|_{\mathbb{H}_{0}^{1}}^{2} \mathrm{~d} t\right] \leq C\left[\mathbb{E}\left\|Y_{T}\right\|_{\mathbb{H}_{0}^{1}}^{2}+\mathbb{E} \int_{0}^{T}\|f(t)\|_{\mathbb{L}^{2}}^{2} \mathrm{~d} t\right] .
$$

The existence of a strong solution to (2.10), as well as its uniqueness are shown in [14]. Obviously, $(Y, Z)$ satisfies the following variational form $\mathbb{P}$-a.s. for all times $t \in[0, T]$

$$
\begin{aligned}
\left(Y_{T}, \phi\right)_{\mathbb{L}^{2}} & -(Y(t), \phi)_{\mathbb{L}^{2}}-\int_{t}^{T}\left[(\nabla Y(s), \nabla \phi)_{\mathbb{L}^{2}}+(f(s), \phi)_{\mathbb{L}^{2}}\right] \mathrm{d} s \\
& =\int_{t}^{T}(Z(s), \phi)_{\mathbb{L}^{2}} \mathrm{~d} W(s) \quad \forall \phi \in \mathbb{H}_{0}^{1} .
\end{aligned}
$$

We now consider a finite element discretization of the BSPDE (2.10). Let $Y_{T, h} \in L_{\mathcal{F}_{T}}^{2}\left(\Omega ; \mathbb{V}_{h}^{1}\right)$ be an approximation of $Y_{T}$. The problem $\mathbf{B S P D E}_{h}$ then reads: Find $\left(Y_{h}, Z_{h}\right) \in L_{\mathbb{F}}^{2}\left(\Omega ; C\left([0, T] ; \mathbb{V}_{h}^{1}\right)\right) \times L_{\mathbb{F}}^{2}\left(\Omega ; L^{2}\left(0, T ; \mathbb{V}_{h}^{1}\right)\right)$ 
such that $\mathbb{P}$-a.s. for all $t \in[0, T]$

$$
\begin{gathered}
\left(Y_{T, h}, \phi_{h}\right)_{\mathbb{L}^{2}}-\left(Y_{h}(t), \phi_{h}\right)_{\mathbb{L}^{2}}-\int_{t}^{T}\left[\left(\nabla Y_{h}(s), \nabla \phi_{h}\right)_{\mathbb{L}^{2}}+\left(f(s), \phi_{h}\right)_{\mathbb{L}^{2}}\right] \mathrm{d} s \\
=\int_{t}^{T}\left(Z_{h}(s), \phi_{h}\right)_{\mathbb{L}^{2}} \mathrm{~d} W(s) \quad \forall \phi_{h} \in \mathbb{V}_{h}^{1} .
\end{gathered}
$$

Equation (2.13) is equivalent to the following BSDE:

$$
\left\{\begin{array}{l}
\mathrm{d} Y_{h}(t)=\left[-\Delta_{h} Y_{h}(t)+\Pi_{h}^{1} f(t)\right] \mathrm{d} t+Z_{h}(t) \mathrm{d} W(t) \quad \forall t \in[0, T] \\
Y_{h}(T)=Y_{T, h}
\end{array}\right.
$$

Based on this equivalence, we do not distinguish $\mathbf{B S P D E}_{h}$ (2.13) and BSDE (2.14) throughout this paper. The existence and uniqueness of a solution tuple $\left(Y_{h}, Z_{h}\right)$ e.g. follows from Theorem 2.1 of [17]. Moreover, there exists $C \equiv C(f, T)>0$ such that

$$
\sup _{t \in[0, T]} \mathbb{E}\left[\left\|\nabla Y_{h}(t)\right\|_{\mathbb{L}^{2}}^{2}\right]+\mathbb{E}\left[\int_{0}^{T}\left\|\Delta_{h} Y_{h}(t)\right\|_{\mathbb{L}^{2}}^{2}+\left\|\nabla Z_{h}(t)\right\|_{\mathbb{L}^{2}}^{2} \mathrm{~d} t\right] \leq C \mathbb{E}\left[\left\|\nabla Y_{T, h}\right\|_{\mathbb{L}^{2}}^{2}+\int_{0}^{T}\|f(t)\|_{\mathbb{L}^{2}}^{2} \mathrm{~d} t\right]
$$

cf. Lemma 3.1 of [15].- The following result is taken from Theorem 3.2 of [15], whose proof exploits the bounds (2.11).

Theorem 2.1. Let $Y_{T} \in L_{\mathcal{F}_{T}}^{2}\left(\Omega ; \mathbb{H}_{0}^{1}\right), Y_{T, h} \in L_{\mathcal{F}_{T}}^{2}\left(\Omega ; \mathbb{V}_{h}^{1}\right)$. Let $(Y, Z)$ be the solution to $(2.12)$, and $\left(Y_{h}, Z_{h}\right)$ solve (2.13). There exists $C \equiv C\left(Y_{T}, f, T\right)>0$ such that

$$
\begin{aligned}
\sup _{t \in[0, T]} & \mathbb{E}\left[\left\|Y(t)-Y_{h}(t)\right\|_{\mathbb{L}^{2}}^{2}\right]+\mathbb{E}\left[\int_{0}^{T}\left\|\nabla\left[Y(t)-Y_{h}(t)\right]\right\|_{\mathbb{L}^{2}}^{2}+\left\|Z(t)-Z_{h}(t)\right\|_{\mathbb{L}^{2}}^{2} \mathrm{~d} t\right] \\
\leq & C\left(\mathbb{E}\left[\left\|Y_{T}-Y_{T, h}\right\|_{\mathbb{L}^{2}}^{2}\right]+h^{2}\right) .
\end{aligned}
$$

Choosing $Y_{T, h}=\Pi_{h}^{1} Y_{T}$ thus leads to an error estimate for the spatial semi-discretization (2.14).

\subsection{Temporal discretization of the backward stochastic heat equation - the role of the Malliavin derivative}

The numerical analysis of a temporal discretization of (2.14) requires Malliavin calculus to bound temporal increments $\mathbb{E}\left[\left\|Z_{h}(t)-Z_{h}(s)\right\|_{\mathbb{L}^{2}}^{2}\right]$ uniformly in $h$ in terms of $|t-s|$, where $s, t \in[0, T]$. We therefore recall the definition of the Malliavin derivative of processes, and the crucial connection between the Malliavin derivative of $Y_{h}$ and $Z_{h}$ from (2.14). For further details, we refer to [17,31].

Let us recall that $\mathcal{F}_{T}=\sigma\{W(t) ; 0 \leq t \leq T\}$, and that $\mathbb{K}$ denotes a separable Hilbert space. We define the Itô isometry $W: L^{2}(0, T ; \mathbb{R}) \rightarrow L_{\mathcal{F}_{T}}^{2}(\Omega ; \mathbb{R})$ by

$$
W(g)=\int_{0}^{T} g(t) \mathrm{d} W(t) .
$$

For $\ell \in \mathbb{N}$, we denote by $C_{p}^{\infty}\left(\mathbb{R}^{\ell}\right)$ the space of all smooth functions $s: \mathbb{R}^{\ell} \rightarrow \mathbb{R}$ such that $s$ and all of its partial derivatives have polynomial growth. Let $\mathcal{P}$ be the set of $\mathbb{R}$-valued random variables of the form

$$
F=s\left(W\left(h_{1}\right), \ldots, W\left(h_{\ell}\right)\right)
$$


for some $s \in C_{p}^{\infty}\left(\mathbb{R}^{\ell}\right), \ell \in \mathbb{N}$, and $g_{1}, \ldots, g_{\ell} \in L^{2}(0, T ; \mathbb{R})$. To any $F \in \mathcal{P}$ we define its $\mathbb{R}$-valued Malliavin derivative $D F:=\left\{D_{\theta} F ; 0 \leq \theta \leq T\right\}$ process via

$$
D_{\theta} F=\sum_{i=1}^{\ell} \frac{\partial s}{\partial x_{i}}\left(W\left(g_{1}\right), \ldots, W\left(g_{\ell}\right)\right) g_{i}(\theta) .
$$

In general, we can define the $k$-th iterated derivative of $F$ by $D^{k} F=D\left(D^{k-1} F\right)$, for any $k \in \mathbb{N}$.

Now we extend the derivative operator to $\mathbb{K}$-valued variables. For any $k \in \mathbb{N}$, and $u$ in the set of $\mathbb{K}$-valued variables:

$$
\mathcal{P}_{\mathbb{K}}=\left\{u=\sum_{j=1}^{n} F_{j} \phi_{j}: F_{j} \in \mathcal{P}, \phi_{j} \in \mathbb{K}, n \in \mathbb{N}\right\},
$$

we can define the $k$-th iterated derivative of $u$ by

$$
D^{k} u=\sum_{j=1}^{n} D^{k} F_{j} \otimes \phi_{j}
$$

For $p \geq 1$, we define the norm $\|\cdot\|_{k, p}$ via

$$
\|u\|_{k, p}:=\left(\mathbb{E}\left[\|u\|_{\mathbb{K}}^{p}+\sum_{j=1}^{k}\left\|D^{j} u\right\|_{\left(L^{2}(0, T ; \mathbb{R})\right)^{\otimes j} \otimes \mathbb{K}}^{p}\right]\right)^{\frac{1}{p}}
$$

Then $\mathbb{D}^{k, p}(\mathbb{K})$ is the completion of $\mathcal{P}_{\mathbb{K}}$ under the norm $\|\cdot\|_{k, p}$.

We may now express $Z_{h}$ in BSDE (2.14) in terms of the Malliavin derivative of $Y_{h}$.

Lemma 2.2 ([17], Prop. 5.3). Suppose that $Y_{T, h} \in \mathbb{D}^{1,2}\left(\mathbb{L}^{2}\right), f \in L_{\mathbb{F}}^{2}\left(\Omega ; L^{2}\left(0, T ; \mathbb{L}^{2}\right)\right)$, and

$$
\mathbb{E}\left[\int_{0}^{T}\left\|D_{\theta} Y_{T, h}\right\|_{\mathbb{L}^{2}}^{2} \mathrm{~d} \theta\right]+\mathbb{E}\left[\int_{0}^{T} \int_{0}^{T}\left\|D_{\theta} f(t)\right\|_{\mathbb{L}^{2}}^{2} \mathrm{~d} t \mathrm{~d} \theta\right]<\infty
$$

Let $\left(Y_{h}, Z_{h}\right)$ be the solution to BSDE (2.14). Then

$$
\left(Y_{h}, Z_{h}\right) \in L_{\mathbb{F}}^{2}\left(\Omega ; C\left([0, T] ; \mathbb{D}^{1,2}\left(\mathbb{L}^{2}\right)\right) \times L^{2}\left(0, T ; \mathbb{D}^{1,2}\left(\mathbb{L}^{2}\right)\right)\right),
$$

and its Malliavin derivative $\left(D_{\theta} Y_{h}, D_{\theta} Z_{h}\right)$ solves

$$
\left\{\begin{array}{c}
D_{\theta} Y_{h}(t)-D_{\theta} Y_{h}(T)+\int_{t}^{T}-\Delta_{h} D_{\theta} Y_{h}(s)+\Pi_{h}^{1} D_{\theta} f(s) \mathrm{d} s \\
=-\int_{t}^{T} D_{\theta} Z_{h}(s) \mathrm{d} W(s) \quad 0 \leq \theta \leq t \leq T, \\
D_{\theta} Y_{h}(t)=D_{\theta} Z_{h}(t)=0 \quad 0 \leq t<\theta \leq T .
\end{array}\right.
$$

Moreover, $\left\{D_{t} Y_{h}(t): 0 \leq t \leq T\right\}$ is a version of $\left\{Z_{h}(t): 0 \leq t \leq T\right\}$. 


\section{Strong RATES of CONVERGEnCE FOR A SPACE-TIME DisCRETIZATION OF THE BSPDE (2.10)}

In this section, we introduce the temporal discretization scheme (3.6) to approximate the solution $\left(Y_{h}, Z_{h}\right)$ to the $\operatorname{BSPDE}_{h}(2.14)$ by a finite sequence $\left\{\left(Y_{h}^{n}, Z_{h}^{n}\right)\right\}_{n=0}^{N-1}$ on a mesh $I_{\tau}$. The main results are Theorems 3.5 and 3.7 in Section 3.2. Their derivation crucially hinges on the time regularity of the solution $\left(Y_{h}, Z_{h}\right)$ to $(2.14)$, and a related uniform bound w.r.t. the mesh parameter $h>0$, which is provided in the subsequent Section 3.1.

\subsection{Uniform bounds for temporal increments of the solution $\left(\boldsymbol{Y}_{\boldsymbol{h}}, \boldsymbol{Z}_{\boldsymbol{h}}\right)$ to $(2.14)$}

We start with the derivation of uniform estimates for $Y_{h}$ which control its temporal increments. We note again that all involved generic constants $C>0$ do not depend on $h$.

Lemma 3.1. Suppose that $Y_{T, h} \in L_{\mathcal{F}_{T}}^{2}\left(\Omega ; \mathbb{H}_{0}^{1}\right), f \in L_{\mathbb{F}}^{2}\left(\Omega ; L^{2}\left(0, T ; \mathbb{H}_{0}^{1}\right)\right), I_{\tau}$ is a temporal partition of $[0, T]$. Let $\left(Y_{h}, Z_{h}\right)$ be the solution to (2.14). Then

(i)

$$
\sum_{n=0}^{N-1} \mathbb{E}\left[\int_{t_{n}}^{t_{n+1}}\left\|Y_{h}(t)-Y_{h}\left(t_{n}\right)\right\|_{\mathbb{L}^{2}}^{2} \mathrm{~d} t\right] \leq C \tau \mathbb{E}\left[\left\|Y_{T, h}\right\|_{\mathbb{H}_{0}^{1}}^{2}+\int_{0}^{T}\|f(t)\|_{\mathbb{L}^{2}}^{2} \mathrm{~d} t\right]
$$

(ii) Assume further $\sup _{h>0} \mathbb{E}\left[\left\|\Delta_{h} Y_{T, h}\right\|_{\mathbb{L}^{2}}^{2}\right]<\infty$. Then

$$
\sum_{n=0}^{N-1} \mathbb{E}\left[\int_{t_{n}}^{t_{n+1}}\left\|\nabla\left(Y_{h}(t)-Y_{h}\left(t_{n}\right)\right)\right\|_{\mathbb{L}^{2}}^{2} \mathrm{~d} t\right] \leq C \tau \mathbb{E}\left[\left\|\Delta_{h} Y_{T, h}\right\|_{\mathbb{L}^{2}}^{2}+\left\|\nabla Y_{T, h}\right\|_{\mathbb{H}_{0}^{1}}^{2}+\int_{0}^{T}\|f(t)\|_{\mathbb{H}_{0}^{1}}^{2} \mathrm{~d} t\right] .
$$

(iii) Assume further $\sup _{h>0} \mathbb{E}\left[\left\|\Delta_{h} Y_{T, h}\right\|_{\mathbb{H}_{0}^{1}}^{2}\right]<\infty$ and $f \in L_{\mathbb{F}}^{2}\left(\Omega ; L^{2}\left(0, T ; \mathbb{H}_{0}^{1} \cap \mathbb{H}^{2}\right)\right)$. Then

$$
\sum_{n=0}^{N-1} \mathbb{E}\left[\int_{t_{n}}^{t_{n+1}}\left\|\Delta_{h}\left(Y_{h}(t)-Y_{h}\left(t_{n}\right)\right)\right\|_{\mathbb{L}^{2}}^{2} \mathrm{~d} t\right] \leq C \tau \mathbb{E}\left[\left\|\Delta_{h} Y_{T, h}\right\|_{\mathbb{H}_{0}^{1}}^{2}+\int_{0}^{T}\|f(t)\|_{\mathbb{H}^{2}}^{2} \mathrm{~d} t\right] .
$$

Here, the constant $C>0$ only depends on $Y_{T, h}, f$ and $T$.

Proof. We only prove (i). The other statements can be proved in a similar vein.

By BSDE (2.14), we get

$$
\sum_{n=0}^{N-1} \mathbb{E}\left[\int_{t_{n}}^{t_{n+1}}\left\|Y_{h}(t)-Y_{h}\left(t_{n}\right)\right\|_{\mathbb{L}^{2}}^{2} \mathrm{~d} t\right] \leq C \tau \mathbb{E}\left[\int_{0}^{T}\left\|\Delta_{h} Y_{h}(t)\right\|_{\mathbb{L}^{2}}^{2}+\left\|\Pi_{h}^{1} f(t)\right\|_{\mathbb{L}^{2}}^{2}+\left\|Z_{h}(t)\right\|_{\mathbb{L}^{2}}^{2} \mathrm{~d} t\right] .
$$

Applying Itô's formula for $\left\|Y_{h}\right\|_{\mathbb{L}^{2}}^{2}$ and $\left\|\nabla Y_{h}\right\|_{\mathbb{L}^{2}}^{2}$ in (2.14), we find that

$$
\begin{aligned}
& \mathbb{E}\left[\int_{0}^{T}\left\|Z_{h}(t)\right\|_{\mathbb{L}^{2}}^{2} \mathrm{~d} t\right] \leq C \mathbb{E}\left[\left\|Y_{T, h}\right\|_{\mathbb{L}^{2}}^{2}+\int_{0}^{T}\left\|\Pi_{h}^{1} f(t)\right\|_{\mathbb{L}^{2}}^{2} \mathrm{~d} t\right], \\
& \mathbb{E}\left[\int_{0}^{T}\left\|\Delta_{h} Y_{h}(t)\right\|_{\mathbb{L}^{2}}^{2} \mathrm{~d} t\right] \leq C \mathbb{E}\left[\left\|\nabla Y_{T, h}\right\|_{\mathbb{L}^{2}}^{2}+\int_{0}^{T}\left\|\Pi_{h}^{1} f(t)\right\|_{\mathbb{L}^{2}}^{2} \mathrm{~d} t\right] .
\end{aligned}
$$

Then (i) can be deduced by the above estimates. 
Lemma 3.2. Suppose that $Y_{T, h} \in \mathbb{D}^{1,2}\left(\mathbb{H}_{0}^{1}\right)$, and $f \in L_{\mathbb{F}}^{2}\left(\Omega ; L^{2}\left(0, T ; \mathbb{L}^{2}\right)\right)$ satisfy

$$
\begin{gathered}
\sup _{0 \leq t \leq T} \mathbb{E}\left[\left\|D_{t} Y_{T, h}\right\|_{\mathbb{H}_{0}^{1}}^{2}\right]+\sup _{0 \leq \theta \leq T} \sup _{0 \leq t \leq T} \mathbb{E}\left[\left\|D_{\theta} D_{t} Y_{T, h}\right\|_{\mathbb{L}^{2}}^{2}\right] \leq C, \\
\sup _{0 \leq t \leq T} \mathbb{E}\left[\int_{t}^{T}\left\|D_{t} f(\tau)\right\|_{\mathbb{L}^{2}}^{2} \mathrm{~d} \tau\right]+\sup _{0 \leq \theta \leq T} \sup _{0 \leq t \leq T} \mathbb{E}\left[\int_{\theta \vee t}^{T}\left\|D_{\theta} D_{t} f(\tau)\right\|_{\mathbb{L}^{2}}^{2} \mathrm{~d} \tau\right] \leq C,
\end{gathered}
$$

and for any $s, t \in[0, T]$ with $s \leq t$,

$$
\mathbb{E}\left[\left\|\left(D_{t}-D_{s}\right) Y_{T, h}\right\|_{\mathbb{L}^{2}}^{2}\right]+\mathbb{E}\left[\int_{t}^{T}\left\|\left(D_{t}-D_{s}\right) f(\tau)\right\|_{\mathbb{L}^{2}}^{2} \mathrm{~d} \tau\right] \leq C|t-s|
$$

Then, it holds that

$$
\mathbb{E}\left[\left\|Z_{h}(t)-Z_{h}(s)\right\|_{\mathbb{L}^{2}}^{2}\right] \leq C|t-s|
$$

Proof. By Lemma 2.2, we know that $Z_{h}(t)=D_{t} Y_{h}(t)$ for all $0 \leq t \leq T$, and therefore, for $0 \leq s \leq t \leq T$,

$$
\frac{1}{2} \mathbb{E}\left[\left\|Z_{h}(t)-Z_{h}(s)\right\|_{\mathbb{L}^{2}}^{2}\right] \leq \mathbb{E}\left[\left\|D_{t} Y_{h}(t)-D_{s} Y_{h}(t)\right\|_{\mathbb{L}^{2}}^{2}\right]+\mathbb{E}\left[\left\|D_{s} Y_{h}(t)-D_{s} Y_{h}(s)\right\|_{\mathbb{L}^{2}}^{2}\right]
$$

In what follows, we estimate the two terms on the right-hand side of (3.2) independently.

Step 1. Fix two $0 \leq \theta_{2} \leq \theta_{1} \leq t \leq T$ and define $\delta_{\theta}=D_{\theta_{1}}-D_{\theta_{2}}$. By (2.17), we have the BSDE

$$
\delta_{\theta} Y_{h}(t)-\delta_{\theta} Y_{h}(T)+\int_{t}^{T}\left[-\Delta_{h} \delta_{\theta} Y_{h}(s)+\Pi_{h}^{1} \delta_{\theta} f(s)\right] \mathrm{d} s=-\int_{t}^{T} \delta_{\theta} Z_{h}(s) \mathrm{d} W(s) \forall t \in\left[\theta_{1}, T\right] .
$$

Itô's formula and Poincaré's inequality lead to

$$
\begin{gathered}
\mathbb{E}\left[\left\|\delta_{\theta} Y_{h}(t)\right\|_{\mathbb{L}^{2}}^{2}\right]+\int_{t}^{T} \mathbb{E}\left[\left\|\nabla \delta_{\theta} Y_{h}(s)\right\|_{\mathbb{L}^{2}}^{2}+\left\|\delta_{\theta} Z_{h}(s)\right\|_{\mathbb{L}^{2}}^{2}\right] \mathrm{d} s \\
\leq \mathbb{E}\left[\left\|\delta_{\theta} Y_{h}(T)\right\|_{\mathbb{L}^{2}}^{2}+\int_{t}^{T}\left\|\Pi_{h}^{1} \delta_{\theta} f(s)\right\|_{\mathbb{L}^{2}}^{2} \mathrm{~d} s\right] .
\end{gathered}
$$

Taking $\theta_{2}=s$ and $\theta_{1}=t$ and using (3.1) then lead to

$$
\mathbb{E}\left[\left\|D_{t} Y_{h}(t)-D_{s} Y_{h}(t)\right\|_{\mathbb{L}^{2}}^{2}\right] \leq \mathbb{E}\left[\left\|\left(D_{t}-D_{s}\right) Y_{h, T}\right\|_{\mathbb{L}^{2}}^{2}+\int_{t}^{T}\left\|\left(D_{t}-D_{s}\right) f(\tau)\right\|_{\mathbb{L}^{2}}^{2} \mathrm{~d} \tau\right] \leq C|t-s|
$$


Step 2. By (2.17), Itô's isometry together with Poincaré's inequality,

$$
\begin{aligned}
& \mathbb{E}\left[\left\|D_{s} Y_{h}(t)-D_{s} Y_{h}(s)\right\|_{\mathbb{L}^{2}}^{2}\right] \\
= & \mathbb{E}\left[\left\|\int_{s}^{t}\left[-\Delta_{h} D_{s} Y_{h}(\tau)+\Pi_{h}^{1} D_{s} f(\tau)\right] \mathrm{d} \tau+\int_{s}^{t} D_{s} Z_{h}(\tau) \mathrm{d} W(\tau)\right\|_{\mathbb{L}^{2}}^{2}\right] \\
\leq & 2|t-s| \int_{s}^{T} \mathbb{E}\left[\left\|\Delta_{h} D_{s} Y_{h}(\tau)\right\|_{\mathbb{L}^{2}}^{2}+\left\|\Pi_{h}^{1} D_{s} f(\tau)\right\|_{\mathbb{L}^{2}}^{2}\right] \mathrm{d} \tau+2 \int_{s}^{t} \mathbb{E}\left[\left\|D_{s} Z_{h}(\tau)\right\|_{\mathbb{L}^{2}}^{2}\right] \mathrm{d} \tau \\
\leq & C|t-s| \mathbb{E}\left[\left\|\nabla D_{s} Y_{h}(T)\right\|_{\mathbb{L}^{2}}^{2}+\int_{s}^{T}\left\|\Pi_{h}^{1} D_{s} f(\tau)\right\|_{\mathbb{L}^{2}}^{2} \mathrm{~d} \tau\right] \\
& +C|t-s| \sup _{0 \leq \theta \leq T} \sup _{0 \leq t \leq T} \mathbb{E}\left[\left\|D_{\theta} D_{t} Y_{T, h}\right\|_{\mathbb{L}^{2}}^{2}+\int_{\theta \vee t}^{T}\left\|D_{\theta} D_{t} f(\tau)\right\|_{\mathbb{L}^{2}}^{2} \mathrm{~d} \tau\right] .
\end{aligned}
$$

Inserting (3.5) and (3.4) into (3.2) then settles the proof of the lemma.

Remark 3.3. In Lemma 3.2, we derive the Malliavin differentiability of the solution to $\mathbf{B S P D E}_{h}(2.14)$, i.e., the spatial discretization for BSPDE (2.10). Indeed, the Malliavin differentiability of solution to (2.10) can also be verified; see e.g. Proposition 3.2 of [12].

\subsection{A time-implicit space-time discretization of the BSPDE (2.10)}

We use a time implicit discretization on the mesh $I_{\tau}$ to approximate $\mathbf{B S P D E}_{h}(2.14)$; we refer to it as $\mathbf{B S P D E}_{h \tau}$, and the discretization reads as follows: For every $0 \leq n \leq N-1$, find $\left(Y_{h}^{n}, Z_{h}^{n}\right) \in L_{\mathcal{F}_{t_{n}}}^{2}\left(\Omega ; \mathbb{V}_{h}^{1} \times \mathbb{V}_{h}^{1}\right)$ such that

$$
\left\{\begin{array}{l}
{\left[\mathbb{1}-\tau \Delta_{h}\right] Y_{h}^{n}=\mathbb{E}\left[Y_{h}^{n+1} \mid \mathcal{F}_{t_{n}}\right]-\tau \Pi_{h}^{1} f\left(t_{n}\right),} \\
Z_{h}^{n}=\frac{1}{\tau} \mathbb{E}\left[Y_{h}^{n+1} \Delta_{n+1} W \mid \mathcal{F}_{t_{n}}\right] \\
Y_{h}^{N}=Y_{T, h}
\end{array}\right.
$$

We introduce an auxiliary BSDE for the convergence analysis of (3.6), which, in particular, uses a time-continuous diffusion term:

$$
\left\{\begin{array}{l}
\mathrm{d} \bar{Y}_{h}(t)=\left[-\Delta_{h} Y_{h}^{\pi(t)}+\Pi_{h}^{1} f(\tau(t))\right] \mathrm{d} t+\bar{Z}_{h}(t) \mathrm{d} W(t) \quad \forall t \in[0, T), \\
\bar{Y}_{h}(T)=Y_{T, h},
\end{array}\right.
$$

where $\pi(\cdot)$, and $\tau(\cdot)$ are defined as follows: For $n=0,1, \ldots, N-1$ and $t \in\left[t_{n}, t_{n+1}\right)$, we set $\pi(t)=n$ and $\tau(t)=t_{n}$.

Lemma 3.4. Let $\left\{\left(Y_{h}^{n}, Z_{h}^{n}\right)\right\}_{n=0}^{N-1}$ solve (3.6), and $\left(\bar{Y}_{h}, \bar{Z}_{h}\right)$ solve (3.7). For all $0 \leq n \leq N-1$,

$$
Y_{h}^{n}=\bar{Y}_{h}\left(t_{n}\right), \quad Z_{h}^{n}=\frac{1}{\tau} \mathbb{E}\left[\int_{t_{n}}^{t_{n+1}} \bar{Z}_{h}(s) \mathrm{d} s \mid \mathcal{F}_{t_{n}}\right] .
$$

Proof. The first identity is immediate; the second follows from multiplication of (3.7) with the admissible $\int_{t_{n}}^{t_{n+1}} 1 \mathrm{~d} W(s)$, and application of conditional expectation $\mathbb{E}\left[\cdot \mid \mathcal{F}_{t_{n}}\right]$.

We may now prove a strong error estimate for the first component of $\left(Y_{h}, Z_{h}\right)$ that solves (2.14); its proof uses the auxiliary BSDE (3.7), and the relation of its solution at time-grid points to iterates $\left\{\left(Y_{h}^{n}, Z_{h}^{n}\right)\right\}_{n=0}^{N-1}$ from (3.6) thanks to Lemma 3.4. 
Theorem 3.5. Suppose that $Y_{T, h} \in L_{\mathcal{F}_{T}}^{2}\left(\Omega ; \mathbb{H}_{0}^{1}\right), \Delta_{h} Y_{T, h} \in L_{\mathcal{F}_{T}}^{2}\left(\Omega ; \mathbb{L}^{2}\right), f \in L_{\mathbb{F}}^{2}\left(\Omega ; L^{2}\left(0, T ; \mathbb{H}_{0}^{1}\right)\right)$ as well as

$$
\sum_{n=0}^{N-1} \int_{t_{n}}^{t_{n+1}} \mathbb{E}\left[\left\|f(t)-f\left(t_{n}\right)\right\|_{\mathbb{L}^{2}}^{2}\right] \mathrm{d} t \leq C \tau
$$

Let $\left(Y_{h}, Z_{h}\right)$ solve (2.14), and $\left\{\left(Y_{h}^{n}, Z_{h}^{n}\right)\right\}_{n=0}^{N-1}$ solve (3.6). There exists a constant $C \equiv\left(Y_{T, h}, f, T\right)>0$ such that

$$
\max _{0 \leq n \leq N} \mathbb{E}\left[\left\|Y_{h}\left(t_{n}\right)-Y_{h}^{n}\right\|_{\mathbb{L}^{2}}^{2}\right]+\tau \sum_{n=0}^{N-1} \mathbb{E}\left[\left\|\nabla\left(Y_{h}\left(t_{n}\right)-Y_{h}^{n}\right)\right\|_{\mathbb{L}^{2}}^{2}\right] \leq C \tau
$$

Proof. Consider $\left(\bar{Y}_{h}, \bar{Z}_{h}\right)$ from (3.7), and define $\left\{e_{n}\right\}_{n=0}^{N-1}$, where each $e_{n}=Y_{h}\left(t_{n}\right)-\bar{Y}_{h}\left(t_{n}\right)$ is a $\mathbb{V}_{h}^{1}$-valued random variable. Subtracting (3.7) from (2.14) yields $\mathbb{P}$-a.s.

$$
\begin{aligned}
e_{n}-e_{n+1}-\int_{t_{n}}^{t_{n+1}} \Delta_{h} e_{n} \mathrm{~d} s= & \int_{t_{n}}^{t_{n+1}} \Delta_{h}\left[Y_{h}(s)-Y_{h}\left(t_{n}\right)\right]-\Pi_{h}^{1}\left[f(s)-f\left(t_{n}\right)\right] \mathrm{d} s \\
& -\int_{t_{n}}^{t_{n+1}}\left[Z_{h}(s)-\bar{Z}_{h}(s)\right] \mathrm{d} W(s) .
\end{aligned}
$$

Fixing one realization $\omega \in \Omega$, testing with the admissible $e_{n}(\omega) \in \mathbb{V}_{h}^{1}$, using binomial formula, and then taking expectation, and Poincare's and Young's inequality lead to

$$
\begin{aligned}
\frac{1}{2} \mathbb{E} & {\left[\left\|e_{n}\right\|_{\mathbb{L}^{2}}^{2}-\left\|e_{n+1}\right\|_{\mathbb{L}^{2}}^{2}+\left\|e_{n}-e_{n+1}\right\|_{\mathbb{L}^{2}}^{2}+2 \int_{t_{n}}^{t_{n+1}}\left\|\nabla e_{n}\right\|_{\mathbb{L}^{2}}^{2} \mathrm{~d} s\right] } \\
\leq & \mathbb{E}\left[\int_{t_{n}}^{t_{n+1}} \frac{1}{2}\left\|\nabla\left[Y_{h}(s)-Y_{h}\left(t_{n}\right)\right]\right\|_{\mathbb{L}^{2}}^{2}+\left\|f(s)-f\left(t_{n}\right)\right\|_{\mathbb{L}^{2}}^{2} \mathrm{~d} s\right] \\
& +\frac{1}{2} \mathbb{E}\left[\int_{t_{n}}^{t_{n+1}}\left\|\nabla e_{n}\right\|_{\mathbb{L}^{2}}^{2}+\left\|e_{n}-e_{n+1}\right\|_{\mathbb{L}^{2}}^{2} \mathrm{~d} s\right]+\frac{\tau}{2} \mathbb{E}\left[\left\|e_{n+1}\right\|_{\mathbb{L}^{2}}\right]^{2} .
\end{aligned}
$$

Subsequently, the discrete Gronwall inequality leads to

$$
\max _{0 \leq n \leq N} \mathbb{E}\left[\left\|e_{n}\right\|_{\mathbb{L}^{2}}^{2}\right] \leq 2 e^{T} \sum_{n=0}^{N-1} \mathbb{E}\left[\int_{t_{n}}^{t_{n+1}}\left\|\nabla\left[Y_{h}(s)-Y_{h}\left(t_{n}\right)\right]\right\|_{\mathbb{L}^{2}}^{2}+\left\|f(s)-f\left(t_{n}\right)\right\|_{\mathbb{L}^{2}}^{2} \mathrm{~d} s\right]
$$

Then, summing up over all steps of (3.10) yields

$$
\begin{aligned}
& \sum_{n=0}^{N-1} \mathbb{E}\left[\int_{t_{n}}^{t_{n+1}}\left\|\nabla e_{n}\right\|_{\mathbb{L}^{2}}^{2} \mathrm{~d} s\right] \\
& \leq \tau \sum_{n=0}^{N-1} \mathbb{E}\left[\left\|e_{n+1}\right\|_{\mathbb{L}^{2}}\right]^{2}+2 \sum_{n=0}^{N-1} \mathbb{E}\left[\int_{t_{n}}^{t_{n+1}}\left\|\nabla\left[Y_{h}(s)-Y_{h}\left(t_{n}\right)\right]\right\|_{\mathbb{L}^{2}}^{2}+\left\|f(s)-f\left(t_{n}\right)\right\|_{\mathbb{L}^{2}}^{2} \mathrm{~d} s\right] .
\end{aligned}
$$

Then, (3.11), (3.12) together with Lemma 3.1 (ii), and Lemma 3.4 lead to the desired estimate. 
By Theorems 2.1, 3.5 and Lemma 3.1 (i), we thus get the following convergence rate for the approximation $\left\{Y_{h}^{n}\right\}_{n=0}^{N}$ of the first solution component $Y$ to (2.12) via the space-time discretization scheme (3.6),

$$
\max _{0 \leq n \leq N} \mathbb{E}\left[\left\|Y\left(t_{n}\right)-Y_{h}^{n}\right\|_{\mathbb{L}^{2}}^{2}\right]+\sum_{n=0}^{N-1} \int_{t_{n}}^{t_{n+1}} \mathbb{E}\left[\left\|\nabla\left(Y(t)-Y_{h}^{n}\right)\right\|_{\mathbb{L}^{2}}^{2}\right] \mathrm{d} t \leq C\left(\tau+h^{2}\right) .
$$

Remark 3.6. If the drift term of $(2.10)$ is $-\Delta Y(t, x)+f(t, x, Y(t, x))$, where $f$ is a Lipschitz nonlinearity, we may apply a similar procedure to get the above convergence rate. However, the above strategy is not clear to be successful if $Z$ appears as well in the drift term.

We now derive estimates for the approximation $\left\{Z_{h}^{n}\right\}_{n=0}^{N-1}$ of the second solution component $Z$ to (2.12), which uses the characterization $Z_{h}(t)=D_{t} Y_{h}(t)$, and $(2.17)$.

Theorem 3.7. Let $\left(Y_{h}, Z_{h}\right)$ solve (2.13), where data satisfy the assumptions in Lemma 3.1 (ii), Lemma 3.2, as well as

$$
\sum_{n=0}^{N-1} \int_{t_{n}}^{t_{n+1}} \mathbb{E}\left[\left\|f(t)-f\left(t_{n}\right)\right\|_{\mathbb{L}^{2}}^{2}\right] \mathrm{d} t \leq C \tau
$$

Let $\left\{\left(Y_{h}^{n}, Z_{h}^{n}\right)\right\}_{n=0}^{N-1}$ solve (3.6). There exists a constant $C \equiv\left(Y_{T}, f, T\right)>0$ such that

$$
\sum_{n=0}^{N-1} \int_{t_{n}}^{t_{n+1}} \mathbb{E}\left[\left\|Z_{h}(t)-Z_{h}^{n}\right\|_{\mathbb{L}^{2}}^{2}\right] \mathrm{d} t \leq C \tau .
$$

The proof begins with an estimate for $Z_{h}-\bar{Z}_{h}$, which exploits time regularity properties of the solution $\left(Y_{h}, Z_{h}\right)$ in stronger norms; $c f$. Lemma 3.1, (ii). Moreover, the following technical result is needed; see also [35].

Lemma 3.8. For any $\varphi \in L_{\mathbb{F}}^{2}(0, T ; \mathbb{K})$ and $0 \leq s<t \leq T$, define

$$
\varphi_{0}=\frac{1}{t-s} \mathbb{E}\left[\int_{s}^{t} \varphi(\tau) \mathrm{d} \tau \mid \mathcal{F}_{s}\right] .
$$

For any $\xi \in L_{\mathcal{F}_{s}}^{2}(\Omega ; \mathbb{K})$ there holds

$$
\mathbb{E}\left[\int_{s}^{t}\left\|\varphi(\tau)-\varphi_{0}\right\|_{\mathbb{K}}^{2} \mathrm{~d} \tau\right] \leq \mathbb{E}\left[\int_{s}^{t}\|\varphi(\tau)-\xi\|_{\mathbb{K}}^{2} \mathrm{~d} \tau\right]
$$

Proof. Let $\left\{\phi_{i}\right\}_{i=1}^{\infty}$ be an orthonormal basis of $\mathbb{K}$, and $\Pi_{n}$ be the projection from $\mathbb{K}$ to $\operatorname{span}\left\{\phi_{i}: i=1,2, \ldots, n\right\}$. For any $n \in \mathbb{Z}$, one has

$$
\begin{aligned}
& \mathbb{E}\left[\int_{s}^{t}\|\varphi(\tau)-\xi\|_{\mathbb{K}}^{2} \mathrm{~d} \tau\right] \geq \mathbb{E}\left[\int_{s}^{t}\left\|\Pi_{n}(\varphi(\tau)-\xi)\right\|_{\mathbb{K}}^{2} \mathrm{~d} \tau\right] \\
=\mathbb{E} & {\left[\int_{s}^{t}\left\|\Pi_{n}\left(\varphi(\tau)-\varphi_{0}\right)\right\|_{\mathbb{K}}^{2}+\left\|\Pi_{n}\left(\varphi_{0}-\xi\right)\right\|_{\mathbb{K}}^{2}+2\left(\Pi_{n}\left(\varphi(\tau)-\varphi_{0}\right), \Pi_{n}\left(\varphi_{0}-\xi\right)\right)_{\mathbb{K}} \mathrm{d} \tau\right] } \\
=\mathbb{E} & {\left[\int_{s}^{t}\left\|\Pi_{n}\left(\varphi(\tau)-\varphi_{0}\right)\right\|_{\mathbb{K}}^{2}+\|\left.\Pi_{n}\left(\varphi_{0}-\xi\right)\right|_{\mathbb{K}} ^{2} \mathrm{~d} \tau\right] } \\
& +2 \mathbb{E}\left[\mathbb{E}\left[\left(\int_{s}^{t} \Pi_{n} \varphi(\tau) \mathrm{d} \tau-\mathbb{E}\left[\int_{s}^{t} \Pi_{n} \varphi(\tau) \mathrm{d} \tau \mid \mathcal{F}_{s}\right], \Pi_{n}\left(\varphi_{0}-\xi\right)\right)_{\mathbb{K}} \mid \mathcal{F}_{s}\right]\right]
\end{aligned}
$$


Since, $\varphi_{0}$ and $\xi$ are $\mathcal{F}_{s}$-measurable, the last term vanishes, i.e.,

$$
\begin{aligned}
\mathbb{E}[\mathbb{E} & {\left.\left[\left(\int_{s}^{t} \Pi_{n} \varphi(\tau) \mathrm{d} \tau-\mathbb{E}\left[\int_{s}^{t} \Pi_{n} \varphi(\tau) \mathrm{d} \tau \mid \mathcal{F}_{s}\right], \Pi_{n}\left(\varphi_{0}-\xi\right)\right)_{\mathbb{K}} \mid \mathcal{F}_{s}\right]\right] } \\
& =\mathbb{E}\left[\left(\mathbb{E}\left[\int_{s}^{t} \Pi_{n} \varphi(\tau) \mathrm{d} \tau-\mathbb{E}\left[\int_{s}^{t} \Pi_{n} \varphi(\tau) \mathrm{d} \tau \mid \mathcal{F}_{s}\right] \mid \mathcal{F}_{s}\right], \Pi_{n}\left(\varphi_{0}-\xi\right)\right)_{\mathbb{K}}\right]=0 .
\end{aligned}
$$

Therefore,

$$
\begin{aligned}
\mathbb{E}\left[\int_{s}^{t}\|\varphi(\tau)-\xi\|_{\mathbb{K}}^{2} \mathrm{~d} \tau\right] & \geq \mathbb{E}\left[\int_{s}^{t}\left\|\Pi_{n}\left(\varphi(\tau)-\varphi_{0}\right)\right\|_{\mathbb{K}}^{2} \mathrm{~d} \tau\right]+\mathbb{E}\left[\int_{s}^{t} \|\left.\Pi_{n}\left(\varphi_{0}-\xi\right)\right|_{\mathbb{K}} ^{2} \mathrm{~d} \tau\right] \\
& \geq \mathbb{E}\left[\int_{s}^{t}\left\|\Pi_{n}\left(\varphi(\tau)-\varphi_{0}\right)\right\|_{\mathbb{K}}^{2} \mathrm{~d} \tau\right] .
\end{aligned}
$$

By letting $n \uparrow \infty$, we may therefore conclude

$$
\mathbb{E}\left[\int_{s}^{t}\left\|\varphi(\tau)-\varphi_{0}\right\|_{\mathbb{K}}^{2} \mathrm{~d} \tau\right]=\lim _{n \rightarrow \infty} \mathbb{E}\left[\int_{s}^{t}\left\|\Pi_{n}\left(\varphi(\tau)-\varphi_{0}\right)\right\|_{\mathbb{K}}^{2} \mathrm{~d} \tau\right] \leq \mathbb{E}\left[\int_{s}^{t}\|\varphi(\tau)-\xi\|_{\mathbb{K}}^{2} \mathrm{~d} \tau\right]
$$

which completes the proof.

Proof of Theorem 3.7. Step 1. Claim: theres exists a constant $C$, which is independent of $h, \tau$, such that

$$
\mathbb{E}\left[\int_{0}^{T}\left\|Z_{h}(s)-\bar{Z}_{h}(s)\right\|_{\mathbb{L}^{2}}^{2} \mathrm{~d} s\right] \leq C \tau
$$

We recall the definition of $\left\{e_{n}\right\}_{n=0}^{N-1}$ in the proof of Theorem 3.5, as well as equation (3.9), which we recast into the form

$$
\begin{aligned}
(\mathbb{1}- & \left.\tau \Delta_{h}\right) e_{n}+\int_{t_{n}}^{t_{n+1}}\left[\bar{Z}_{h}(s)-Z_{h}(s)\right] \mathrm{d} W(s) \\
& =e_{n+1}+\int_{t_{n}}^{t_{n+1}}\left[\Delta_{h}\left(Y_{h}(s)-Y_{h}\left(t_{n}\right)\right)-\Pi_{h}^{1}\left(f(s)-f\left(t_{n}\right)\right)\right] \mathrm{d} s .
\end{aligned}
$$

Taking squares and afterwards expectations on both sides, by binomial formula, Itô isometry, and Young's inequality, we arrive at

$$
\begin{aligned}
\mathbb{E} & {\left[\left\|\left(\mathbb{1}-\tau \Delta_{h}\right) e_{n}\right\|_{\mathbb{L}^{2}}^{2}+\left\|\int_{t_{n}}^{t_{n+1}} \bar{Z}_{h}(s)-Z_{h}(s) \mathrm{d} W(s)\right\|_{\mathbb{L}^{2}}^{2}\right] } \\
& =\mathbb{E}\left[\left\|\left(\mathbb{1}-\tau \Delta_{h}\right) e_{n}\right\|_{\mathbb{L}^{2}}^{2}+\int_{t_{n}}^{t_{n+1}}\left\|\bar{Z}_{h}(s)-Z_{h}(s)\right\|_{\mathbb{L}^{2}}^{2} \mathrm{~d} s\right] \\
& \leq(1+\tau) \mathbb{E}\left[\left\|e_{n+1}\right\|_{\mathbb{L}^{2}}^{2}+\left(1+\frac{1}{4 \tau}\right) \tau \int_{t_{n}}^{t_{n+1}}\left\|\Delta_{h}\left[Y_{h}(s)-Y_{h}\left(t_{n}\right)\right]-\Pi_{h}^{1}\left[f(s)-f\left(t_{n}\right)\right]\right\|_{\mathbb{L}^{2}}^{2} \mathrm{~d} s\right] .
\end{aligned}
$$


Note that $\left\|\left(\mathbb{1}-\tau \Delta_{h}\right) e_{n}\right\|_{\mathbb{L}^{2}}^{2}=\left\|e_{n}\right\|_{\mathbb{L}^{2}}^{2}+2 \tau\left\|\nabla e_{n}\right\|_{\mathbb{L}^{2}}^{2}+\tau^{2}\left\|\Delta_{h} e_{n}\right\|_{\mathbb{L}^{2}}^{2}$. Summation over $0 \leq n \leq N-1$ then leads to

$$
\begin{aligned}
& \mathbb{E}\left[\left\|e_{0}\right\|_{\mathbb{L}^{2}}^{2}+2 \tau \sum_{n=0}^{N-1}\left\|\nabla e_{n}\right\|_{\mathbb{L}^{2}}^{2}+\int_{0}^{T}\left\|\bar{Z}_{h}(s)-Z_{h}(s)\right\|_{\mathbb{L}^{2}}^{2} \mathrm{~d} s\right] \\
& \quad \leq \tau \sum_{n=0}^{N-1} \mathbb{E}\left[\left\|e_{n+1}\right\|_{\mathbb{L}^{2}}^{2}\right]+2 \sum_{n=0}^{N-1} \mathbb{E}\left[\int_{t_{n}}^{t_{n+1}}\left\|\Delta_{h}\left[Y_{h}(s)-Y_{h}\left(t_{n}\right)\right]-\Pi_{h}^{1}\left[f(s)-f\left(t_{n}\right)\right]\right\|_{\mathbb{L}^{2}}^{2} \mathrm{~d} s\right] .
\end{aligned}
$$

By the discrete version of Gronwall's inequality, and Lemma 3.1, (iii), the right-hand side is bounded by $C \tau$. Hence, (3.14) is proved.

Step 2. We use the triangle inequality, Lemma 3.4 and Lemma 3.8 with $\xi=Z_{h}(\tau(t))$, as well as Lemma 3.2 , to deduce

$$
\begin{aligned}
& \sum_{n=0}^{N-1} \mathbb{E}\left[\int_{t_{n}}^{t_{n+1}}\left\|Z_{h}(t)-Z_{h}^{n}\right\|_{\mathbb{L}^{2}}^{2} \mathrm{~d} t\right] \\
& \quad \leq 2 \sum_{n=0}^{N-1} \int_{t_{n}}^{t_{n+1}} \mathbb{E}\left[\left\|Z_{h}(t)-\bar{Z}_{h}(t)\right\|_{\mathbb{L}^{2}}^{2}+\left\|\bar{Z}_{h}(t)-Z_{h}^{n}\right\|_{\mathbb{L}^{2}}^{2}\right] \mathrm{d} t \\
& \quad \leq 2 \sum_{n=0}^{N-1} \int_{t_{n}}^{t_{n+1}} \mathbb{E}\left[3\left\|Z_{h}(t)-\bar{Z}_{h}(t)\right\|_{\mathbb{L}^{2}}^{2}+2\left\|Z_{h}(t)-Z_{h}\left(t_{n}\right)\right\|_{\mathbb{L}^{2}}^{2}\right] \mathrm{d} t \\
& \quad \leq C \tau .
\end{aligned}
$$

That completes the proof.

\section{Strong RAtes of CONVERGEnCE FOR A SPACE-TIME Discretization OF SLQ}

In this part, we discretize the original problem SLQ within two steps, starting with its semi-discretization in space (which is referred to as $\mathbf{S} \mathbf{L} \mathbf{Q}_{h}$ ), which is then followed by a discretization in space and time (which is referred to as $\mathbf{S L Q}_{h \tau}$ ). Our goal is to prove strong convergence rates in both cases. By e.g. [25], problem SLQ is uniquely solvable, and its solution $\left(X^{*}, U^{*}\right)$ may be characterized by the following FBSPDE with the unique solution $\left(X^{*}, Y, Z, U^{*}\right)$,

$$
\left\{\begin{array}{l}
\mathrm{d} X^{*}(t)=\left[\Delta X^{*}(t)+U^{*}(t)\right] \mathrm{d} t+\sigma(t) \mathrm{d} W(t) \quad \forall t \in(0, T), \\
\mathrm{d} Y(t)=\left[-\Delta Y(t)+\left[X^{*}(t)-\tilde{X}(t)\right]\right] \mathrm{d} t+Z(t) \mathrm{d} W(t) \quad \forall t \in(0, T), \\
X^{*}(0)=X_{0}, \quad Y(T)=-\alpha\left(X^{*}(T)-\tilde{X}(T)\right),
\end{array}\right.
$$

with the condition

$$
U^{*}-Y=0 .
$$

We remark that by $(4.1)_{1}, X^{*}$ may be written as $X^{*}=\mathcal{S}\left(U^{*}\right)$, where

$$
\mathcal{S}: L_{\mathbb{F}}^{2}\left(\Omega ; L^{2}\left(0, T ; \mathbb{L}^{2}\right)\right) \rightarrow L_{\mathbb{F}}^{2}\left(\Omega ; C\left([0, T] ; \mathbb{H}_{0}^{1}\right) \cap L^{2}\left(0, T ; \mathbb{H}^{2}\right)\right)
$$


is the bounded 'control-to-state' map. Moreover, we introduce the reduced functional

$$
\widehat{\mathcal{J}}: L_{\mathbb{F}}^{2}\left(\Omega ; L^{2}\left(0, T ; \mathbb{L}^{2}\right)\right) \rightarrow \mathbb{R} \quad \text { via } \quad \widehat{\mathcal{J}}(U)=\mathcal{J}(\mathcal{S}(U), U)
$$

where $\mathcal{J}$ is defined in (1.1). The first component of the solution to equation $(4.1)_{2}$ may be written as $Y=\mathcal{T}\left(X^{*}\right)$, where $\mathcal{T}$

$$
\mathcal{T}: L_{\mathbb{F}}^{2}\left(\Omega ; C\left([0, T] ; \mathbb{L}^{2}\right)\right) \rightarrow L_{\mathbb{F}}^{2}\left(\Omega ; C\left([0, T] ; \mathbb{H}_{0}^{1}\right) \cap L^{2}\left(0, T ; \mathbb{H}_{0}^{1} \cap \mathbb{H}^{2}\right)\right)
$$

which is also bounded. For every $U \in L_{\mathbb{F}}^{2}\left(\Omega ; L^{2}\left(0, T ; \mathbb{L}^{2}\right)\right)$, the Fréchet derivative $D \widehat{\mathcal{J}}(U)$ is also a bounded operator on $L_{\mathbb{F}}^{2}\left(\Omega ; L^{2}\left(0, T ; \mathbb{L}^{2}\right)\right)$ and takes the form

$$
D \widehat{\mathcal{J}}(U)=U-\mathcal{T}(\mathcal{S}(U))
$$

\subsection{Problem $\mathrm{SLQ}_{h}$ : Semi-discretization in space}

We begin with a spatial semi-discretization $\mathbf{S L Q}_{h}$ of the problem SLQ stated in the introduction, which reads: Find an optimal pair $\left(X_{h}^{*}, U_{h}^{*}\right) \in L_{\mathbb{F}}^{2}\left(\Omega ; C\left([0, T] ; \mathbb{V}_{h}^{1}\right) \times L^{2}\left(0, T ; \mathbb{V}_{h}^{0}\right)\right)$ that minimizes the functional $(\alpha \geq 0)$

$$
\mathcal{J}\left(X_{h}, U_{h}\right)=\frac{1}{2} \mathbb{E}\left[\int_{0}^{T}\left\|X_{h}(t)-\widetilde{X}(t)\right\|_{\mathbb{L}^{2}}^{2}+\left\|U_{h}(t)\right\|_{\mathbb{L}^{2}}^{2} \mathrm{~d} t+\alpha\left\|X_{h}(T)-\widetilde{X}(T)\right\|_{\mathbb{L}^{2}}^{2}\right]
$$

subject to the equation

$$
\left\{\begin{array}{l}
\mathrm{d} X_{h}(t)=\left[\Delta_{h} X_{h}(t)+\Pi_{h}^{1} U_{h}(t)\right] \mathrm{d} t+\Pi_{h}^{1} \sigma(t) \mathrm{d} W(t) \quad \forall t \in[0, T] \\
X_{h}(0)=\Pi_{h}^{1} X_{0}
\end{array}\right.
$$

The existence of a unique optimal pair $\left(X_{h}^{*}, U_{h}^{*}\right)$ follows from [38], as well as its characterization via Pontryagin's maximum principle, i.e.,

$$
0=U_{h}^{*}(t)-\Pi_{h}^{0} Y_{h}(t) \quad \forall t \in(0, T),
$$

where the adjoint $\left(Y_{h}, Z_{h}\right) \in L_{\mathbb{F}}^{2}\left(\Omega ; C\left([0, T] ; \mathbb{V}_{h}^{1}\right)\right) \times L_{\mathbb{F}}^{2}\left(\Omega ; L^{2}\left(0, T ; \mathbb{V}_{h}^{1}\right)\right)$ solves the $\mathbf{B S P D E}_{h}$

$$
\left\{\begin{array}{l}
\mathrm{d} Y_{h}(t)=\left[-\Delta_{h} Y_{h}(t)+\left[X_{h}^{*}(t)-\Pi_{h}^{1} \widetilde{X}(t)\right]\right] \mathrm{d} t+Z_{h}(t) \mathrm{d} W(t) \quad \forall t \in[0, T] \\
Y_{h}(T)=-\alpha\left(X_{h}^{*}(T)-\Pi_{h}^{1} \widetilde{X}(T)\right) .
\end{array}\right.
$$

In [15], optimal error estimates have been obtained for $\left(X_{h}^{*}, Y_{h}, Z_{h}\right)$ with the help of a fixed point argumentwhich crucially exploits $T>0$ to be sufficiently small. The goal in this section is to derive corresponding estimates for $\left(X_{h}^{*}, U_{h}^{*}, Y_{h}, Z_{h}\right)$ for arbitrary $T>0$ via a variational argument which exploits properties of the

reduced functional $\widehat{\mathcal{J}} \equiv \widehat{\mathcal{J}}(u)$ that is now defined: once an estimate for $\int_{0}^{T} \mathbb{E}\left[\left\|U^{*}(s)-U_{h}^{*}(s)\right\|_{\mathbb{L}^{2}}^{2}\right] \mathrm{d} s$ has been obtained, we use the convergence analysis from Section 3 to derive estimates for $X^{*}-X_{h}^{*}$, as well as $Y-Y_{h}$ and $Z-Z_{h}$.

By the unique solvability property of (4.5), we associate to this equation the bounded solution operator

$$
\mathcal{S}_{h}: L_{\mathbb{F}}^{2}\left(\Omega ; L^{2}\left(0, T ; \mathbb{V}_{h}^{0}\right)\right) \rightarrow L_{\mathbb{F}}^{2}\left(\Omega ; C\left([0, T] ; \mathbb{V}_{h}^{1}\right)\right),
$$


which allows to introduce the reduced functional

$$
\widehat{\mathcal{J}}_{h}: L_{\mathbb{F}}^{2}\left(\Omega ; L^{2}\left(0, T ; \mathbb{V}_{h}^{0}\right)\right) \rightarrow \mathbb{R}, \quad \text { via } \quad \widehat{\mathcal{J}}_{h}\left(U_{h}\right)=\mathcal{J}\left(\mathcal{S}_{h}\left(U_{h}\right), U_{h}\right),
$$

where $\mathcal{J}$ is defined in (1.1). The first solution component to equation (4.7) may be written as $Y_{h}=\mathcal{T}_{h}\left(X_{h}^{*}\right)$, where

$$
\mathcal{T}_{h}: L_{\mathbb{F}}^{2}\left(\Omega ; C\left([0, T] ; \mathbb{V}_{h}^{1}\right)\right) \rightarrow L_{\mathbb{F}}^{2}\left(\Omega ; C\left([0, T] ; \mathbb{V}_{h}^{1}\right)\right)
$$

For every $U_{h} \in L_{\mathbb{F}}^{2}\left(\Omega ; L^{2}\left(0, T ; \mathbb{V}_{h}^{0}\right)\right)$, the Fréchet derivative $D \widehat{\mathcal{J}}_{h}\left(U_{h}\right)$ is a bounded operator (uniformly in $h$ ) on $L_{\mathbb{F}}^{2}\left(\Omega ; L^{2}\left(0, T ; \mathbb{V}_{h}^{0}\right)\right)$ at $U_{h}$, and has the form

$$
D \widehat{\mathcal{J}}_{h}\left(U_{h}\right)=U_{h}-\Pi_{h}^{0} \mathcal{T}_{h}\left(\mathcal{S}_{h}\left(U_{h}\right)\right)
$$

Let $U_{h} \in L_{\mathbb{F}}^{2}\left(\Omega ; L^{2}\left(0, T ; \mathbb{V}_{h}^{0}\right)\right)$ be arbitrary; it is due to the quadratic structure of the reduced functional (4.8) that

$$
\mathbb{E}\left[\left(D^{2} \widehat{\mathcal{J}}_{h}\left(U_{h}\right) R_{h}, R_{h}\right)_{L^{2}\left(0, T ; \mathbb{L}^{2}\right)}\right] \geq \mathbb{E}\left[\left\|R_{h}\right\|_{L^{2}\left(0, T ; \mathbb{L}^{2}\right)}^{2}\right] \quad \forall R_{h} \in L_{\mathbb{F}}^{2}\left(\Omega ; L^{2}\left(0, T ; \mathbb{V}_{h}^{0}\right)\right)
$$

As a consequence, on putting $R_{h}=U_{h}^{*}-\Pi_{h}^{0} U^{*}$,

$$
\begin{aligned}
& \mathbb{E}\left[\left\|U_{h}^{*}-\Pi_{h}^{0} U^{*}\right\|_{L^{2}\left(0, T ; \mathbb{L}^{2}\right)}^{2}\right] \leq \mathbb{E}\left[\left(D^{2} \widehat{\mathcal{J}}_{h}\left(U_{h}\right)\left(U_{h}^{*}-\Pi_{h}^{0} U^{*}\right), U_{h}^{*}-\Pi_{h}^{0} U^{*}\right)_{L^{2}\left(0, T ; \mathbb{L}^{2}\right)}\right] \\
& =\mathbb{E}\left[\left(D \widehat{\mathcal{J}}_{h}\left(U_{h}^{*}\right), U_{h}^{*}-\Pi_{h}^{0} U^{*}\right)_{L^{2}\left(0, T ; \mathbb{L}^{2}\right)}-\left(D \widehat{\mathcal{J}}_{h}\left(\Pi_{h}^{0} U^{*}\right), U_{h}^{*}-\Pi_{h}^{0} U^{*}\right)_{L^{2}\left(0, T ; \mathbb{L}^{2}\right)}\right] .
\end{aligned}
$$

Note that $D \widehat{\mathcal{J}}_{h}\left(U_{h}^{*}\right)=0$ by (4.6), as well as $D \widehat{\mathcal{J}}\left(U^{*}\right)=0$ by (4.2), such that the last line equals

$$
\begin{aligned}
= & \mathbb{E}\left[\left(D \widehat{\mathcal{J}}\left(U^{*}\right), U_{h}^{*}-\Pi_{h}^{0} U^{*}\right)_{L^{2}\left(0, T ; \mathbb{L}^{2}\right)}-\left(D \widehat{\mathcal{J}}\left(\Pi_{h}^{0} U^{*}\right), U_{h}^{*}-\Pi_{h}^{0} U^{*}\right)_{L^{2}\left(0, T ; \mathbb{L}^{2}\right)}\right] \\
& +\mathbb{E}\left[\left(D \widehat{\mathcal{J}}\left(\Pi_{h}^{0} U^{*}\right), U_{h}^{*}-\Pi_{h}^{0} U^{*}\right)_{L^{2}\left(0, T ; \mathbb{L}^{2}\right)}-\left(D \widehat{\mathcal{J}}_{h}\left(\Pi_{h}^{0} U^{*}\right), U_{h}^{*}-\Pi_{h}^{0} U^{*}\right)_{L^{2}\left(0, T ; \mathbb{L}^{2}\right)}\right] \\
= & : I+I I .
\end{aligned}
$$

We use (4.3) to bound $I$ as follows,

$$
\begin{aligned}
I & =\mathbb{E}\left[\left(U^{*}-\Pi_{h}^{0} U^{*}+\mathcal{T}\left(\mathcal{S}\left(\Pi_{h}^{0} U^{*}\right)\right)-\mathcal{T}\left(\mathcal{S}\left(U^{*}\right)\right), U_{h}^{*}-\Pi_{h}^{0} U^{*}\right)_{L^{2}\left(0, T ; \mathbb{L}^{2}\right)}\right] \\
& \leq\left(\left(\mathbb{E}\left[\left\|U^{*}-\Pi_{h}^{0} U^{*}\right\|_{L^{2}\left(0, T ; \mathbb{L}^{2}\right)}^{2}\right]\right)^{1 / 2}+I_{a}\right)\left(\mathbb{E}\left[\left\|U_{h}^{*}-\Pi_{h}^{0} U^{*}\right\|_{L^{2}\left(0, T ; \mathbb{L}^{2}\right)}^{2}\right]\right)^{1 / 2}
\end{aligned}
$$

where $I_{a}^{2}=\mathbb{E}\left[\left\|\mathcal{T}\left(\mathcal{S}\left(U^{*}\right)-\mathcal{S}\left(\Pi_{h}^{0} U^{*}\right)\right)\right\|_{L^{2}\left(0, T ; \mathbb{L}^{2}\right)}^{2}\right.$. By Poincaré's inequality, and a stability bound (see also (2.11)) for the backward stochastic heat equation (2.10), as well as for the stochastic heat equation (2.3) (see also $(2.2))$,

$$
\begin{aligned}
I_{a}^{2} & \leq C \mathbb{E}\left[\left\|\left(\mathcal{S}\left(U^{*}\right)-\mathcal{S}\left(\Pi_{h}^{0} U^{*}\right)\right)(T)\right\|_{\mathbb{L}^{2}}^{2}+\left\|\mathcal{S}\left(U^{*}\right)-\mathcal{S}\left(\Pi_{h}^{0} U^{*}\right)\right\|_{L^{2}\left(0, T ; \mathbb{L}^{2}\right)}^{2}\right] \\
& \leq C \mathbb{E}\left[\left\|U^{*}-\Pi_{h}^{0} U^{*}\right\|_{L^{2}\left(0, T ; \mathbb{L}^{2}\right)}^{2}\right] .
\end{aligned}
$$


By optimality condition (4.2), and the regularity properties of the solution to BSPDE (2.10), we know that already $U^{*} \in L_{\mathbb{F}}^{2}\left(\Omega ; C\left([0, T] ; \mathbb{H}_{0}^{1}\right) \cap L^{2}\left(0, T ; \mathbb{H}_{0}^{1} \cap \mathbb{H}^{2}\right)\right)$; as a consequence, the right-hand side of (4.12) may be bounded by $C h^{2}$.

We use the representation (4.9) and properties of the projection $\Pi_{h}^{0}$ to bound $I I$ via

$$
\begin{aligned}
I I & =\mathbb{E}\left[\left(\mathcal{T}\left(\mathcal{S}\left(\Pi_{h}^{0} U^{*}\right)\right)-\Pi_{h}^{0} \mathcal{T}_{h}\left(\mathcal{S}_{h}\left(\Pi_{h}^{0} U^{*}\right)\right), U_{h}^{*}-\Pi_{h}^{0} U^{*}\right)_{L^{2}\left(0, T ; \mathbb{L}^{2}\right)}\right] \\
& \leq I I_{a} \times\left(\mathbb{E}\left[\left\|U_{h}^{*}-\Pi_{h}^{0} U^{*}\right\|_{L^{2}\left(0, T ; \mathbb{L}^{2}\right)}^{2}\right]\right)^{1 / 2},
\end{aligned}
$$

where $I I_{a}^{2}:=\mathbb{E}\left[\left\|\mathcal{T}\left(\mathcal{S}\left(\Pi_{h}^{0} U^{*}\right)\right)-\mathcal{T}_{h}\left(\mathcal{S}_{h}\left(\Pi_{h}^{0} U^{*}\right)\right)\right\|_{L^{2}\left(0, T ; \mathbb{L}^{2}\right)}^{2}\right]$. We split $I I_{a}^{2}$ into two terms

$$
\begin{aligned}
I I_{a, 1}^{2} & =\mathbb{E}\left[\left\|\mathcal{T}\left(\mathcal{S}\left(\Pi_{h}^{0} U^{*}\right)\right)-\mathcal{T}\left(\mathcal{S}_{h}\left(\Pi_{h}^{0} U^{*}\right)\right)\right\|_{L^{2}\left(0, T ; \mathbb{L}^{2}\right)}^{2}\right] \\
\text { and } \quad I I_{a, 2}^{2} & =\mathbb{E}\left[\left\|\mathcal{T}\left(\mathcal{S}_{h}\left(\Pi_{h}^{0} U^{*}\right)\right)-\mathcal{T}_{h}\left(\mathcal{S}_{h}\left(\Pi_{h}^{0} U^{*}\right)\right)\right\|_{L^{2}\left(0, T ; \mathbb{L}^{2}\right)}^{2}\right]
\end{aligned}
$$

In order to bound $I I_{a, 1}^{2}$, we use stability properties for BSPDE (2.10), in combination with the error estimate (2.6) for (2.5) to conclude

$$
I I_{a, 1}^{2} \leq C \mathbb{E}\left[\left\|\mathcal{S}\left(\Pi_{h}^{0} U^{*}\right)-\mathcal{S}_{h}\left(\Pi_{h}^{0} U^{*}\right)\right\|_{L^{2}\left(0, T ; \mathbb{L}^{2}\right)}^{2}\right] \leq C h^{2}
$$

In order to bound $I I_{a, 2}^{2}$, we use the error estimate in Theorem 2.1 for BSPDE (2.10), in combination with stability properties of (2.5), and again the error estimate (2.6) for (2.5) to find

$$
I I_{a, 2}^{2} \leq C\left(\mathbb{E}\left[\left\|\mathcal{S}\left(\Pi_{h}^{0} U^{*}\right)(T)-\mathcal{S}_{h}\left(\Pi_{h}^{0} U^{*}\right)(T)\right\|_{\mathbb{L}^{2}}^{2}\right]+h^{2}\right) \leq C h^{2} .
$$

We now insert these estimates into (4.11) resp. (4.10) to obtain the bound

$$
\mathbb{E}\left[\left\|U_{h}^{*}-\Pi_{h}^{0} U^{*}\right\|_{L^{2}\left(0, T ; \mathbb{L}^{2}\right)}^{2}\right] \leq C h^{2} .
$$

By arguing as below (4.12), this settles part (i) of the following

Theorem 4.1. Let $\left(X^{*}, Y, Z, U^{*}\right)$ be the solution to problem SLQ, and $\left(X_{h}^{*}, Y_{h}, Z_{h}, U_{h}^{*}\right)$ be the solution to problem $\mathbf{S L Q}_{h}$. There exists $C \equiv C\left(X_{0}, T\right)>0$ such that

$$
\begin{aligned}
& \text { (i) } \mathbb{E}\left[\int_{0}^{T}\left\|U^{*}(t)-U_{h}^{*}(t)\right\|_{\mathbb{L}^{2}}^{2} \mathrm{~d} t\right] \leq C h^{2} ; \\
& \text { (ii) } \sup _{0 \leq t \leq T} \mathbb{E}\left[\left\|X^{*}(t)-X_{h}^{*}(t)\right\|_{\mathbb{L}^{2}}^{2}\right]+\int_{0}^{T} \mathbb{E}\left[\left\|X^{*}(t)-X_{h}^{*}(t)\right\|_{\mathbb{H}_{0}^{1}}^{2}\right] \mathrm{d} t \leq C h^{2} ; \\
& \text { (iii) } \sup _{0 \leq t \leq T} \mathbb{E}\left[\left\|Y(t)-Y_{h}(t)\right\|_{\mathbb{L}^{2}}^{2}\right]+\int_{0}^{T} \mathbb{E}\left[\left\|Y(t)-Y_{h}(t)\right\|_{\mathbb{H}_{0}^{1}}^{2}+\left\|Z(t)-Z_{h}(t)\right\|_{\mathbb{L}^{2}}^{2}\right] \mathrm{d} t \leq C h^{2} .
\end{aligned}
$$

Proof. Since $U^{*} \in L_{\mathbb{F}}^{2}\left(\Omega ; L^{2}\left(0, T ; \mathbb{H}_{0}^{1}\right)\right)$, and (i), the first estimate of (ii) can be deduced as (2.6). Assertion (iii) now follows accordingly as Theorem 2.1 , thanks to (ii).

\subsection{Problem $\mathrm{SLQ}_{h \tau}$ : Discretization in space and time}

In this part, we provide the temporal discretization of problem $\mathbf{S L Q}_{h}$ which was analyzed in Section 4.1. For this purpose, we use a mesh $I_{\tau}$ covering $[0, T]$, and consider processes $\left(X_{h \tau}, U_{h \tau}\right) \in \mathbb{X}_{h \tau} \times \mathbb{U}_{h \tau} \subset$ 
$L_{\mathbb{F}}^{2}\left(\Omega ; L^{2}\left(0, T ; \mathbb{V}_{h}^{1}\right)\right) \times L_{\mathbb{F}}^{2}\left(\Omega ; L^{2}\left(0, T ; \mathbb{V}_{h}^{0}\right)\right)$, where

$$
\begin{aligned}
& \mathbb{X}_{h \tau}:=\left\{X \in L_{\mathbb{F}}^{2}\left(\Omega ; L^{2}\left(0, T ; \mathbb{V}_{h}^{1}\right)\right): X(t)=X\left(t_{n}\right), \forall t \in\left[t_{n}, t_{n+1}\right), n=0,1, \ldots, N-1\right\}, \\
& \mathbb{U}_{h \tau}:=\left\{U \in L_{\mathbb{F}}^{2}\left(\Omega ; L^{2}\left(0, T ; \mathbb{V}_{h}^{0}\right)\right): U(t)=U\left(t_{n}\right), \forall t \in\left[t_{n}, t_{n+1}\right), n=0,1, \ldots, N-1\right\},
\end{aligned}
$$

and for any $X \in \mathbb{X}_{h \tau}, U \in \mathbb{U}_{h \tau}$,

$$
\|X\|_{\mathbb{X}_{h \tau}}:=\left(\tau \sum_{n=1}^{N} \mathbb{E}\left[\left\|X\left(t_{n}\right)\right\|_{\mathbb{L}^{2}}^{2}\right]\right)^{1 / 2},\|U\|_{\mathbb{U}_{h \tau}}:=\left(\tau \sum_{n=0}^{N-1} \mathbb{E}\left[\left\|U\left(t_{n}\right)\right\|_{\mathbb{L}^{2}}^{2}\right]\right)^{1 / 2} .
$$

Problem $\mathbf{S L Q}_{h \tau}$ then reads as follows: Find an optimal pair $\left(X_{h \tau}^{*}, U_{h \tau}^{*}\right) \in \mathbb{X}_{h \tau} \times \mathbb{U}_{h \tau}$ which minimizes the cost functional

$$
\mathcal{J}_{\tau}\left(X_{h \tau}, U_{h \tau}\right)=\frac{1}{2}\left\|X_{h \tau}-\tilde{X}\right\|_{\mathbb{X}_{h \tau}}^{2}+\frac{1}{2}\left\|U_{h \tau}\right\|_{\mathbb{U}_{h \tau}}^{2}+\frac{\alpha}{2} \mathbb{E}\left[\left\|X_{h \tau}(T)-\tilde{X}(T)\right\|_{\mathbb{L}^{2}}^{2}\right],
$$

subject to the difference equation

$$
\left\{\begin{array}{l}
X_{h \tau}\left(t_{n+1}\right)-X_{h \tau}\left(t_{n}\right)=\tau\left[\Delta_{h} X_{h \tau}\left(t_{n+1}\right)+\Pi_{h}^{1} U_{h \tau}\left(t_{n}\right)\right]+\Pi_{h}^{1} \sigma\left(t_{n}\right) \Delta_{n+1} W \quad n=0,1, \cdots, N-1, \\
X_{h \tau}(0)=\Pi_{h}^{1} X_{0},
\end{array}\right.
$$

where $\Delta_{n+1} W=W\left(t_{n+1}\right)-W\left(t_{n}\right)$. The following result states Pontryagin's maximum principle for problem $\mathbf{S L Q} \mathbf{Q}_{h \tau}$, which is later used to verify convergence rates for the solution to problem $\mathbf{S L Q} \mathbf{Q}_{h \tau}$ towards the solution to SLQ.

Theorem 4.2. Problem $\mathbf{S L Q}_{h \tau}$ admits a unique minimizer $\left(X_{h \tau}^{*}, U_{h \tau}^{*}\right) \in \mathbb{X}_{h \tau} \times \mathbb{U}_{h \tau}$, which is (part of) the unique solution

$$
\left(X_{h \tau}^{*}, Y_{h \tau}, U_{h \tau}^{*}\right) \in\left[\mathbb{X}_{h \tau}\right]^{2} \times \mathbb{U}_{h \tau}
$$

to the following forward-backward difference equation for $0 \leq n \leq N-1$,

$$
\left\{\begin{array}{l}
{\left[\mathbb{1}-\tau \Delta_{h}\right] X_{h \tau}^{*}\left(t_{n+1}\right)=X_{h \tau}^{*}\left(t_{n}\right)+\tau \Pi_{h}^{1} U_{h \tau}^{*}\left(t_{n}\right)+\Pi_{h}^{1} \sigma\left(t_{n}\right) \Delta_{n+1} W,} \\
{\left[\mathbb{1}-\tau \Delta_{h}\right] Y_{h \tau}\left(t_{n}\right)=\mathbb{E}\left[Y_{h \tau}\left(t_{n+1}\right)-\tau\left(X_{h \tau}^{*}\left(t_{n+1}\right)-\Pi_{h}^{1} \widetilde{X}\left(t_{n+1}\right)\right) \mid \mathcal{F}_{t_{n}}\right],} \\
X_{h \tau}^{*}(0)=\Pi_{h}^{1} X_{0}, \quad Y_{h \tau}(T)=-\alpha\left(X_{h \tau}^{*}(T)-\Pi_{h}^{1} \widetilde{X}(T)\right),
\end{array}\right.
$$

together with

$$
U_{h \tau}^{*}\left(t_{n}\right)-\Pi_{h}^{0} Y_{h \tau}\left(t_{n}\right)=0 \quad n=0,1, \ldots, N-1 .
$$

By (4.16), we can see that $U_{h \tau}^{*}$ is càdlàg, and then $U_{h \tau}^{*} \in \mathbb{U}_{h \tau}$. Inserting (4.16) into (4.15) 1 leads to a coupled problem for $\left(\left\{X_{h \tau}^{*}\left(t_{n+1}\right)\right\}_{n=0}^{N-1},\left\{Y_{h \tau}\left(t_{n}\right)\right\}_{n=0}^{N-1}\right)$, where $(4.15)_{2}$ is similar to (3.6). Note that no $Z$-component appears explicitly in $(4.15)_{2}$, where the conditional expectation is used to compute the $Y$-component. It is in particular due to the need to compute conditional expectations in $(4.15)_{2}$ that the optimality system (4.15)(4.16) is still not amenable to an actual implementation, but serves as a key step towards a practical method which approximately solves $\mathbf{S L Q} \mathbf{Q}_{h \tau}$-which is proposed and studied in Section 5.

Proof. We divide the proof into three steps. 
Step 1. Let $A_{0}:=\left(\mathbb{1}-\tau \Delta_{h}\right)^{-1}$. For any $U_{h \tau} \in \mathbb{U}_{h \tau}$, by equation $(4.15)_{1}$, we have

$$
X_{h \tau}\left(t_{n}\right)=A_{0}\left[X_{h \tau}\left(t_{n-1}\right)+\tau \Pi_{h}^{1} U_{h \tau}\left(t_{n-1}\right)+\Pi_{h}^{1} \sigma\left(t_{n-1}\right) \Delta_{n} W\right]
$$

Hence, by iteration we arrive at

$$
\begin{aligned}
X_{h \tau}\left(t_{n}\right) & =A_{0}^{n} X_{h \tau}(0)+\tau \sum_{j=0}^{n-1} A_{0}^{n-j} \Pi_{h}^{1} U_{h \tau}\left(t_{j}\right)+\sum_{j=1}^{n} A_{0}^{n+1-j} \Pi_{h}^{1} \sigma\left(t_{j-1}\right) \Delta_{j} W \\
& =:\left(\Gamma \Pi_{h}^{1} X_{0}\right)\left(t_{n}\right)+\left(L U_{h \tau}\right)\left(t_{n}\right)+f\left(t_{n}\right) .
\end{aligned}
$$

Here, $\Gamma: \mathbb{V}_{h}^{1} \rightarrow \mathbb{X}_{h \tau}$ and $L: \mathbb{U}_{h \tau} \rightarrow \mathbb{X}_{h \tau}$ are bounded operators. Below, we use the abbreviations

$$
\widehat{\Gamma} \Pi_{h}^{1} X_{0}:=\Gamma \Pi_{h}^{1} X_{0}(T), \quad \widehat{L} U_{h \tau}:=\left(L U_{h \tau}\right)(T), \quad \widehat{f}=f(T) .
$$

Claim: For any $\xi \in \mathbb{X}_{h \tau}$, and any $\eta \in L_{\mathcal{F}_{T}}^{2}\left(\Omega ; \mathbb{V}_{h}^{1}\right)$,

$$
L^{*} \xi=-\Pi_{h}^{0} Y_{0}, \quad \widehat{L}^{*} \eta=-\Pi_{h}^{0} Y_{1}
$$

where $\left(Y_{0}, Z_{0}\right)$ solves the following backward stochastic difference equation:

$$
\left\{\begin{array}{l}
Y_{0}\left(t_{n+1}\right)-Y_{0}\left(t_{n}\right)=\tau\left[-\Delta_{h} Y_{0}\left(t_{n}\right)+\xi\left(t_{n+1}\right)\right]+\int_{t_{n}}^{t_{n+1}} Z_{0}(t) \mathrm{d} W(t) \quad n=0,1, \ldots, N-1, \\
Y_{0}\left(t_{N}\right)=Y_{0}(T)=0
\end{array}\right.
$$

and $\left(Y_{1}, Z_{1}\right)$ solves

$$
\left\{\begin{array}{l}
Y_{1}\left(t_{n+1}\right)-Y_{1}\left(t_{n}\right)=-\tau \Delta_{h} Y_{1}\left(t_{n}\right)+\int_{t_{n}}^{t_{n+1}} Z_{1}(t) \mathrm{d} W(t) \quad n=0,1, \ldots, N-1, \\
Y_{1}(T)=-\eta
\end{array}\right.
$$

Proof of Claim: The existence and the uniqueness of solutions to (4.21) are obvious. Note that

$$
Y_{0}\left(t_{j}\right)=\mathbb{E}\left[A_{0} Y_{0}\left(t_{j+1}\right)-\tau A_{0} \xi\left(t_{j+1}\right) \mid \mathcal{F}_{t_{j}}\right]
$$

With the similar procedure as that in (4.17), we conclude from (4.22) and $(4.21)_{2}$,

$$
\begin{aligned}
Y_{0}\left(t_{j}\right) & =\mathbb{E}\left[A_{0}^{N-j} Y_{0}\left(t_{N}\right) \mid \mathcal{F}_{t_{j}}\right]-\mathbb{E}\left[\tau \sum_{k=j+1}^{N} A_{0}^{k-j} \xi\left(t_{k}\right) \mid \mathcal{F}_{t_{j}}\right] \\
& =-\mathbb{E}\left[\tau \sum_{k=j+1}^{N} A_{0}^{k-j} \xi\left(t_{k}\right) \mid \mathcal{F}_{t_{j}}\right] .
\end{aligned}
$$


Let $U_{h \tau} \in \mathbb{U}_{h \tau}$ be arbitrary. By the definition of $L,(4.23)$ and the fact that $A_{0}$ is self-adjoint, we can calculate that

$$
\begin{aligned}
\tau \sum_{n=1}^{N} \mathbb{E}\left[\left(\left(L U_{h \tau}\right)\left(t_{n}\right), \xi\left(t_{n}\right)\right)_{\mathbb{L}^{2}}\right]=\tau \sum_{n=1}^{N} \mathbb{E}\left[\left(\tau \sum_{j=0}^{n-1} A_{0}^{n-j} \Pi_{h}^{1} U_{h \tau}\left(t_{j}\right), \xi\left(t_{n}\right)\right)_{\mathbb{L}^{2}}\right] \\
=\tau \sum_{j=0}^{N-1} \mathbb{E}\left[\left(\Pi_{h}^{1} U_{h \tau}\left(t_{j}\right), \mathbb{E}\left[\tau \sum_{n=j+1}^{N} A_{0}^{n-j} \xi\left(t_{n}\right) \mid \mathcal{F}_{t_{j}}\right]\right)_{\mathbb{L}^{2}}\right] .
\end{aligned}
$$

Since the second argument is $\mathbb{V}_{h}^{1}$-valued, we may skip the projection operator in the first argument, and may continue instead

$$
=\tau \sum_{j=0}^{N-1} \mathbb{E}\left[\left(U_{h \tau}\left(t_{j}\right), \Pi_{h}^{0} \mathbb{E}\left[\tau \sum_{k=j+1}^{N} A_{0}^{k-j} \xi\left(t_{k}\right) \mid \mathcal{F}_{t_{j}}\right]\right)_{\mathbb{L}^{2}}\right] .
$$

Because of (4.23), the latter equals

$$
=\tau \sum_{j=0}^{N-1} \mathbb{E}\left[\left(U_{h \tau}\left(t_{j}\right),-\Pi_{h}^{0} Y_{0}\left(t_{j}\right)\right)_{\mathbb{L}^{2}}\right],
$$

which is the first part of the claim.

The remaining part can be deduced from the fact that $Y_{1}\left(t_{j}\right)=-\mathbb{E}\left[A_{0}^{N-j} \eta \mid \mathcal{F}_{t_{j}}\right]$ for $j=0,1, \ldots, N-1$, and the following calculation:

$$
\begin{aligned}
\mathbb{E}\left[\left(\widehat{L} U_{h \tau}, \eta\right)_{\mathbb{L}^{2}}\right] & =\mathbb{E}\left[\left(\tau \sum_{j=0}^{N-1} A_{0}^{N-j} \Pi_{h}^{1} U_{h \tau}\left(t_{j}\right), \eta\right)_{\mathbb{L}^{2}}\right] \\
& =\tau \sum_{j=0}^{N-1} \mathbb{E}\left[\left(\Pi_{h}^{1} U_{h \tau}\left(t_{j}\right), \mathbb{E}\left[A_{0}^{N-j} \eta \mid \mathcal{F}_{t_{j}}\right]\right)_{\mathbb{L}^{2}}\right] \\
& =\tau \sum_{j=0}^{N-1} \mathbb{E}\left[\left(U_{h \tau}\left(t_{j}\right),-\Pi_{h}^{0} Y_{1}\left(t_{j}\right)\right)_{\mathbb{L}^{2}}\right] \quad \forall U_{h \tau} \in \mathbb{U}_{h \tau}
\end{aligned}
$$

Step 2. By (4.18) and (4.19), we can rewrite $\mathcal{J}_{\tau}\left(X_{h \tau}, U_{h \tau}\right)$ as follows:

$$
\begin{aligned}
\mathcal{J}_{\tau}\left(X_{h \tau}, U_{h \tau}\right) & \\
=\frac{1}{2}[ & \left.\left\|X_{h \tau}-\Pi_{\tau} \widetilde{X}\right\|_{L_{\mathbb{F}}^{2}\left(\Omega ; L^{2}\left(0, T ; \mathbb{L}^{2}\right)\right)}^{2}+\left\|U_{h \tau}\right\|_{L_{\mathbb{F}}^{2}\left(\Omega ; L^{2}\left(0, T ; \mathbb{L}^{2}\right)\right)}^{2}+\alpha\left\|X_{h \tau}(T)-\widetilde{X}(T)\right\|_{L_{\mathcal{F}_{T}}^{2}\left(\Omega ; \mathbb{L}^{2}\right)}^{2}\right] \\
=\frac{1}{2}[ & \left(\Gamma \Pi_{h}^{1} X_{0}+L U_{h \tau}+f-\Pi_{\tau} \widetilde{X}, \Gamma \Pi_{h}^{1} X_{0}+L U_{h \tau}+f-\Pi_{\tau} \widetilde{X}\right)_{L_{\mathbb{F}}^{2}\left(\Omega ; L^{2}\left(0, T ; \mathbb{L}^{2}\right)\right)} \\
& \quad+\left(U_{h \tau}, U_{h \tau}\right)_{L_{\mathbb{F}}^{2}\left(\Omega ; L^{2}\left(0, T ; \mathbb{L}^{2}\right)\right)} \\
& \left.+\alpha\left(\widehat{\Gamma} \Pi_{h}^{1} X_{0}+\widehat{L} U_{h \tau}+\widehat{f}-\widetilde{X}(T), \widehat{\Gamma} \Pi_{h}^{1} X_{0}+\widehat{L} U_{h \tau}+\widehat{f}-\widetilde{X}(T)\right)_{L_{\mathcal{F}_{T}}^{2}\left(\Omega ; \mathbb{L}^{2}\right)}\right],
\end{aligned}
$$


where $\Pi_{\tau} \tilde{X}(t)=\tilde{X}\left(t_{n}\right)$, for $t \in\left[t_{n}, t_{n+1}\right), n=0,1, \ldots, N-1$. Rearranging terms then leads to

$$
\begin{aligned}
= & \frac{1}{2}\left[\left(\left[\mathbb{1}+L^{*} L+\alpha \widehat{L}^{*} \widehat{L}\right] U_{h \tau}, U_{h \tau}\right)_{L_{\mathbb{F}}^{2}\left(\Omega ; L^{2}\left(0, T ; \mathbb{L}^{2}\right)\right)}\right. \\
& +2\left(\left[L^{*} \Gamma+\alpha \widehat{L}^{*} \widehat{\Gamma}\right] \Pi_{h}^{1} X_{0}+L^{*} f+\alpha \widehat{L}^{*} \widehat{f}-L^{*} \Pi_{\tau} \widetilde{X}-\alpha \widehat{L}^{*} \widetilde{X}(T), U_{h \tau}\right)_{L_{\mathbb{F}}^{2}\left(\Omega ; L^{2}\left(0, T ; \mathbb{L}^{2}\right)\right)} \\
& +\left\{\left(\Gamma \Pi_{h}^{1} X_{0}+f-\Pi_{\tau} \widetilde{X}, \Gamma \Pi_{h}^{1} X_{0}+f-\Pi_{\tau} \widetilde{X}\right)_{L_{\mathbb{F}}^{2}\left(\Omega ; L^{2}\left(0, T ; \mathbb{L}^{2}\right)\right)}\right. \\
& \left.\left.+\alpha\left(\widehat{\Gamma} \Pi_{h}^{1} X_{0}+\widehat{f}-\widetilde{X}(T), \widehat{\Gamma} \Pi_{h}^{1} X_{0}+\widehat{f}-\widetilde{X}(T)\right)_{L_{\mathcal{F}_{T}}^{2}\left(\Omega ; \mathbb{L}^{2}\right)}\right\}\right] \\
= & : \frac{1}{2}\left[\left(\mathfrak{N} U_{h \tau}, U_{h \tau}\right)_{L_{\mathbb{P}}^{2}\left(\Omega ; L^{2}\left(0, T ; \mathbb{L}^{2}\right)\right)}+2\left(\mathfrak{H}\left(\Pi_{h}^{1} X_{0}, f, \widetilde{X}\right), U_{h \tau}\right)_{L_{\mathbb{F}}^{2}\left(\Omega ; L^{2}\left(0, T ; \mathbb{L}^{2}\right)\right)}+\mathfrak{M}\left(\Pi_{h}^{1} X_{0}, f, \widetilde{X}\right)\right] .
\end{aligned}
$$

Since $\mathfrak{N}=\mathbb{1}+L^{*} L+\alpha \widehat{L}^{*} \widehat{L}$ is positive definite, there exists a unique $U_{h \tau}^{*} \in \mathbb{U}_{h \tau}$ such that

$$
\mathfrak{N} U_{h \tau}^{*}+\mathfrak{H}\left(\Pi_{h}^{1} X_{0}, f, \tilde{X}\right)=0 .
$$

Therefore, for any $U_{h \tau} \in \mathbb{U}_{h \tau}$ such that $U_{h \tau} \neq U_{h \tau}^{*}$,

$$
\begin{aligned}
\mathcal{J}_{\tau} & \left(X_{h \tau}, U_{h \tau}\right)-\mathcal{J}_{\tau}\left(X_{h \tau}^{*}, U_{h \tau}^{*}\right) \\
= & \left(\mathfrak{N} U_{h \tau}^{*}+\mathfrak{H}\left(\Pi_{h}^{1} X_{0}, f, \widetilde{X}\right), U_{h \tau}-U_{h \tau}^{*}\right)_{L_{\mathbb{F}}^{2}\left(\Omega ; L^{2}\left(0, T ; \mathbb{L}^{2}\right)\right)} \\
& +\frac{1}{2}\left(\mathfrak{N}\left(U_{h \tau}-U_{h \tau}^{*}\right), U_{h \tau}-U_{h \tau}^{*}\right)_{L_{\mathbb{P}}^{2}\left(\Omega ; L^{2}\left(0, T ; \mathbb{L}^{2}\right)\right)} \\
= & \frac{1}{2}\left(\mathfrak{N}\left(U_{h \tau}-U_{h \tau}^{*}\right), U_{h \tau}-U_{h \tau}^{*}\right)_{L_{\mathbb{F}}^{2}\left(\Omega ; L^{2}\left(0, T ; \mathbb{L}^{2}\right)\right)} \\
> & 0
\end{aligned}
$$

which means that $U_{h \tau}^{*}$ is the unique optimal control, and $\left(X_{h \tau}^{*}, U_{h \tau}^{*}\right)$ is the unique optimal pair.

Step 3. By the definition of $\mathfrak{N}, \mathfrak{H}, L^{*}, \widehat{L}^{*}$, and properties (4.20) and (4.18), we can get

$$
\begin{aligned}
0 & =\mathfrak{N} U_{h \tau}^{*}+\mathfrak{H}\left(\Pi_{h}^{1} X_{0}, f, \widetilde{X}\right) \\
& =U_{h \tau}^{*}+L^{*}\left(\Gamma \Pi_{h}^{1} X_{0}+L U_{h \tau}^{*}+f-\widetilde{X}\right)+\alpha \widehat{L}^{*}\left(\widehat{\Gamma} \Pi_{h}^{1} X_{0}+\widehat{L} U_{h \tau}^{*}+\widehat{f}-\widetilde{X}(T)\right) \\
& =U_{h \tau}^{*}-\Pi_{h}^{0}\left[Y_{0}\left(\cdot ; X_{h \tau}^{*}-\widetilde{X}\right)+Y_{1}\left(\cdot ; \alpha\left(X_{h \tau}^{*}(T)-\widetilde{X}(T)\right)\right)\right] \\
& =U_{h \tau}^{*}-\Pi_{h}^{0} Y_{h \tau}
\end{aligned}
$$

which is (4.16). This completes the proof.

We are now ready to verify strong rates of convergence for the solution to $\mathbf{S L Q} \mathbf{Q}_{h \tau}$; it is as in Section 4.1 that the reduced cost functional $\widehat{\mathcal{J}}_{h \tau}: \mathbb{U}_{h \tau} \rightarrow \mathbb{R}$ is used, which is defined via

$$
\widehat{\mathcal{J}}_{h \tau}\left(U_{h \tau}\right)=\mathcal{J}_{\tau}\left(\mathcal{S}_{h \tau}\left(U_{h \tau}\right), U_{h \tau}\right),
$$

where $\mathcal{S}_{h \tau}: \mathbb{U}_{h \tau} \rightarrow \mathbb{X}_{h \tau}$ is the solution operator to the forward equation (4.15) $)_{1}$. Moreover, we use the solution operator $\mathcal{T}_{h \tau}: \mathbb{X}_{h \tau} \rightarrow \mathbb{X}_{h \tau}$ for the first solution component of the backward equation (4.15) 2 .

Theorem 4.3. Suppose that $X(0) \in \mathbb{H}_{0}^{1} \cap \mathbb{H}^{2}$, and

$$
\sum_{n=0}^{N-1} \int_{t_{n}}^{t_{n+1}} \mathbb{E}\left[\left\|\sigma(t)-\sigma\left(t_{n}\right)\right\|_{\mathbb{H}_{0}^{1}}^{2}\right]+\left\|\widetilde{X}(t)-\tilde{X}\left(t_{n}\right)\right\|_{\mathbb{L}^{2}}^{2}+\left\|\tilde{X}(t)-\tilde{X}\left(t_{n+1}\right)\right\|_{\mathbb{L}^{2}}^{2} \mathrm{~d} t \leq C \tau .
$$


Let $\left(X_{h}^{*}, Y_{h}, Z_{h}, U_{h}^{*}\right)$ be the solution to problem $\mathbf{S L Q} \mathbf{Q}_{h}$, and $\left(X_{h \tau}^{*}, Y_{h \tau}, U_{h \tau}^{*}\right)$ be the solution to problem $\mathbf{S L Q} \mathbf{Q}_{h \tau}$. There exists $C \equiv C\left(X_{0}, T\right)>0$ such that
(i) $\sum_{k=0}^{N-1} \mathbb{E}\left[\int_{t_{k}}^{t_{k+1}}\left\|U_{h}^{*}(t)-U_{h \tau}^{*}\left(t_{k}\right)\right\|_{\mathbb{L}^{2}}^{2} \mathrm{~d} t\right] \leq C \tau$;
(ii) $\max _{0 \leq k \leq N} \mathbb{E}\left[\left\|X_{h}^{*}\left(t_{k}\right)-X_{h \tau}^{*}\left(t_{k}\right)\right\|_{\mathbb{L}^{2}}^{2}\right]+\mathbb{E}\left[\tau \sum_{k=1}^{N}\left\|X_{h}^{*}\left(t_{k}\right)-X_{h \tau}^{*}\left(t_{k}\right)\right\|_{\mathbb{H}_{0}^{1}}^{2}\right] \leq C \tau$;
(iii) $\max _{0 \leq k \leq N} \mathbb{E}\left[\left\|Y_{h}\left(t_{k}\right)-Y_{h \tau}\left(t_{k}\right)\right\|_{\mathbb{L}^{2}}^{2}\right]+\mathbb{E}\left[\tau \sum_{k=0}^{N-1}\left\|Y_{h}\left(t_{k}\right)-Y_{h \tau}\left(t_{k}\right)\right\|_{\mathbb{H}_{0}^{1}}^{2}\right] \leq C \tau$.

Proof. We divide the proof into three steps.

Step 1. We follow the argumentation in the proof of Theorem 4.1. For every $U_{h \tau}, R_{h \tau} \in \mathbb{U}_{h \tau}$, the first Fréchet derivative $D \widehat{\mathcal{J}}_{h \tau}\left(U_{h \tau}\right)$, and the second Fréchet derivative $D^{2} \widehat{\mathcal{J}}_{h \tau}\left(U_{h \tau}\right)$ satisfy

$$
\begin{aligned}
& D \widehat{\mathcal{J}}_{h \tau}\left(U_{h \tau}\right)=U_{h \tau}-\Pi_{h}^{0} \mathcal{T}_{h \tau}\left(\mathcal{S}_{h \tau}\left(U_{h \tau}\right)\right), \\
& \mathbb{E}\left[\left(D^{2} \widehat{\mathcal{J}}_{h \tau}\left(U_{h \tau}\right) R_{h \tau}, R_{h \tau}\right)_{L^{2}\left(0, T ; \mathbb{L}^{2}\right)}\right] \geq \mathbb{E}\left[\left\|R_{h \tau}\right\|_{L^{2}\left(0, T ; \mathbb{L}^{2}\right)}^{2}\right]
\end{aligned}
$$

Define the (piecewise constant) operator $\Pi_{\tau}: L_{\mathbb{F}}^{2}\left(\Omega ; C\left(0, T ; \mathbb{V}_{h}^{0}\right)\right) \rightarrow \mathbb{U}_{h \tau}$ by

$$
\Pi_{\tau} U_{h}(t):=U_{h}\left(t_{n}\right) \quad \forall t \in\left[t_{n}, t_{n+1}\right) \quad n=0,1, \ldots, N-1 .
$$

By putting $R_{h \tau}=U_{h \tau}^{*}-\Pi_{\tau} U_{h}^{*}$ in (4.26), and applying the fact $D \widehat{\mathcal{J}}_{h \tau}\left(U_{h \tau}^{*}\right)=D \widehat{\mathcal{J}}_{h}\left(U_{h}^{*}\right)=0$, we see that

$$
\begin{aligned}
\mathbb{E} & {\left[\left\|U_{h \tau}^{*}-\Pi_{\tau} U_{h}^{*}\right\|_{L^{2}\left(0, T ; \mathbb{L}^{2}\right)}^{2}\right] } \\
\leq & \mathbb{E}\left[\left(D \widehat{\mathcal{J}}_{h \tau}\left(U_{h \tau}^{*}\right), U_{h \tau}^{*}-\Pi_{\tau} U_{h}^{*}\right)_{L^{2}\left(0, T ; \mathbb{L}^{2}\right)}-\left(D \widehat{\mathcal{J}}_{h \tau}\left(\Pi_{\tau} U_{h}^{*}\right), U_{h \tau}^{*}-\Pi_{\tau} U_{h}^{*}\right)_{L^{2}\left(0, T ; \mathbb{L}^{2}\right)}\right] \\
= & \mathbb{E}\left[\left(D \widehat{\mathcal{J}}_{h}\left(U_{h}^{*}\right)-D \widehat{\mathcal{J}}_{h}\left(\Pi_{\tau} U_{h}^{*}\right), U_{h \tau}^{*}-\Pi_{\tau} U_{h}^{*}\right)_{L^{2}\left(0, T ; \mathbb{L}^{2}\right)}\right] \\
& +\mathbb{E}\left[\left(D \widehat{\mathcal{J}}_{h}\left(\Pi_{\tau} U_{h}^{*}\right)-D \widehat{\mathcal{J}}_{h \tau}\left(\Pi_{\tau} U_{h}^{*}\right), U_{h \tau}^{*}-\Pi_{\tau} U_{h}^{*}\right)_{L^{2}\left(0, T ; \mathbb{L}^{2}\right)}\right] .
\end{aligned}
$$

Therefore,

$$
\begin{aligned}
& \mathbb{E}\left[\left\|U_{h \tau}^{*}-\Pi_{\tau} U_{h}^{*}\right\|_{L^{2}\left(0, T ; \mathbb{L}^{2}\right)}^{2}\right] \\
& \quad \leq 2 \mathbb{E}\left[\left\|D \widehat{\mathcal{J}}_{h}\left(U_{h}^{*}\right)-D \widehat{\mathcal{J}}_{h}\left(\Pi_{\tau} U_{h}^{*}\right)\right\|_{L^{2}\left(0, T ; \mathbb{L}^{2}\right)}^{2}\right]+2 \mathbb{E}\left[\left\|D \widehat{\mathcal{J}}_{h}\left(\Pi_{\tau} U_{h}^{*}\right)-D \widehat{\mathcal{J}}_{h \tau}\left(\Pi_{\tau} U_{h}^{*}\right)\right\|_{L^{2}\left(0, T ; \mathbb{L}^{2}\right)}^{2}\right] \\
& \quad=: 2 I^{\prime}+2 I I^{\prime} .
\end{aligned}
$$

We use (4.9) and (4.6), and stability properties of the projection $\Pi_{h}^{0}$ to bound $I^{\prime}$ as follows,

$$
\begin{aligned}
I^{\prime} & =\mathbb{E}\left[\left\|U_{h}^{*}-\Pi_{\tau} U_{h}^{*}+\Pi_{h}^{0} \mathcal{T}_{h}\left(\mathcal{S}_{h}\left(\Pi_{\tau} U_{h}^{*}\right)\right)-\Pi_{h}^{0} \mathcal{T}_{h}\left(\mathcal{S}_{h}\left(U_{h}^{*}\right)\right)\right\|_{L^{2}\left(0, T ; \mathbb{L}^{2}\right)}^{2}\right] \\
& \leq 2 \mathbb{E}\left[\left\|U_{h}^{*}-\Pi_{\tau} U_{h}^{*}\right\|_{L^{2}\left(0, T ; \mathbb{L}^{2}\right)}^{2}+\left\|\mathcal{T}_{h}\left(\mathcal{S}_{h}\left(\Pi_{\tau} U_{h}^{*}\right)\right)-\mathcal{T}_{h}\left(\mathcal{S}_{h}\left(U_{h}^{*}\right)\right)\right\|_{L^{2}\left(0, T ; \mathbb{L}^{2}\right)}^{2}\right]
\end{aligned}
$$


By stability properties of solutions to $\mathbf{B S P D E}_{h}$ (2.14), and the discretization (2.5) of BSPDE, we obtain

$$
\begin{aligned}
\mathbb{E} & {\left[\left\|\mathcal{T}_{h}\left(\mathcal{S}_{h}\left(\Pi_{\tau} U_{h}^{*}\right)\right)-\mathcal{T}_{h}\left(\mathcal{S}_{h}\left(U_{h}^{*}\right)\right)\right\|_{L^{2}\left(0, T ; \mathbb{L}^{2}\right)}^{2}\right] } \\
& \leq C \mathbb{E}\left[\left\|\left(\mathcal{S}_{h}\left(U_{h}^{*}\right)-\mathcal{S}_{h}\left(\Pi_{\tau} U_{h}^{*}\right)\right)(T)\right\|_{\mathbb{L}^{2}}^{2}+\left\|\mathcal{S}_{h}\left(U_{h}^{*}\right)-\mathcal{S}_{h}\left(\Pi_{\tau} U_{h}^{*}\right)\right\|_{L^{2}\left(0, T ; \mathbb{L}^{2}\right)}^{2}\right] \\
& \leq C\left\|U_{h}^{*}-\Pi_{\tau} U_{h}^{*}\right\|_{L_{\mathbb{P}}^{2}\left(\Omega ; L^{2}\left(0, T ; \mathbb{L}^{2}\right)\right)}^{2} .
\end{aligned}
$$

By the optimality condition (4.6), estimate (2.15), and Theorem 4.1 (i) we have

$$
\begin{aligned}
& \left\|U_{h}^{*}-\Pi_{\tau} U_{h}^{*}\right\|_{L_{\mathbb{F}}^{2}\left(\Omega ; L^{2}\left(0, T ; \mathbb{L}^{2}\right)\right)}^{2} \\
& \quad \leq C \sum_{k=0}^{N-1} \int_{t_{k}}^{t_{k+1}} \int_{t_{k}}^{t} \mathbb{E}\left[\tau\left\|-\Delta_{h} Y_{h}(s)+\left(X_{h}^{*}(s)-\Pi_{h}^{1} \widetilde{X}(s)\right)\right\|_{\mathbb{L}^{2}}^{2}+\left\|Z_{h}(s)\right\|_{\mathbb{L}^{2}}^{2}\right] \mathrm{d} s \mathrm{~d} t \\
& \quad \leq C \tau \int_{0}^{T} \mathbb{E}\left[\left\|-\Delta_{h} Y_{h}(s)+\left(X_{h}^{*}(s)-\Pi_{h}^{1} \widetilde{X}(s)\right)\right\|_{\mathbb{L}^{2}}^{2}+\left\|Z_{h}(s)\right\|_{\mathbb{L}^{2}}^{2}\right] \mathrm{d} s \\
& \quad \leq C \tau .
\end{aligned}
$$

Next, we turn to $I I^{\prime}$, for which we use the representations $(4.9),(4.26)$ and the stability property of $\Pi_{h}^{0}$ to conclude

$$
\begin{aligned}
I I^{\prime}= & \mathbb{E}\left[\left\|\Pi_{h}^{0} \mathcal{T}_{h}\left(\mathcal{S}_{h}\left(\Pi_{\tau} U_{h}^{*}\right)\right)-\Pi_{h}^{0} \mathcal{T}_{h \tau}\left(\mathcal{S}_{h \tau}\left(\Pi_{\tau} U_{h}^{*}\right)\right)\right\|_{L^{2}\left(0, T ; \mathbb{L}^{2}\right)}^{2}\right] \\
\leq & 2 \mathbb{E}\left[\left\|\mathcal{T}_{h}\left(\mathcal{S}_{h}\left(\Pi_{\tau} U_{h}^{*}\right)\right)-\mathcal{T}_{h}\left(\mathcal{S}_{h \tau}\left(\Pi_{\tau} U_{h}^{*}\right)\right)\right\|_{L^{2}\left(0, T ; \mathbb{L}^{2}\right)}^{2}\right. \\
& +\| \mathcal{T}_{h}\left(\mathcal{S}_{h \tau}\left(\Pi_{\tau} U_{h}^{*}\right)\right)-\mathcal{T}_{h \tau}\left(\mathcal{S}_{h \tau}\left(\Pi_{\tau} U_{h}^{*}\right) \|_{L^{2}\left(0, T ; \mathbb{L}^{2}\right)}^{2}\right] \\
= & : 2\left(I I_{a, 1}^{\prime}+I I_{a, 2}^{\prime}\right) .
\end{aligned}
$$

In order to bound $I I_{a, 1}^{\prime}$, we use stability properties for $\mathbf{S P D E}_{h}(2.5), \mathbf{B S P D E}_{h}(4.7)$, in combination with the error estimate (2.8) for (2.5) to conclude

$$
I I_{a, 1}^{\prime} \leq C \mathbb{E}\left[\left\|\left(\mathcal{S}_{h}\left(\Pi_{\tau} U_{h}^{*}\right)-\mathcal{S}_{h \tau}\left(\Pi_{\tau} U_{h}^{*}\right)\right)(T)\right\|_{\mathbb{L}^{2}}^{2}+\left\|\mathcal{S}_{h}\left(\Pi_{\tau} U_{h}^{*}\right)-\mathcal{S}_{h \tau}\left(\Pi_{\tau} U_{h}^{*}\right)\right\|_{L^{2}\left(0, T ; \mathbb{L}^{2}\right)}^{2}\right] \leq C \tau .
$$

To bound $I I_{a, 2}^{\prime}$, it is easy to see

$$
\begin{aligned}
I I_{a, 2}^{\prime} \leq & 2 \sum_{n=0}^{N-1} \mathbb{E}\left[\int_{t_{n}}^{t_{n+1}}\left\|Y_{h}\left(t ; \mathcal{S}_{h \tau}\left(\Pi_{\tau} U_{h}^{*}\right)\right)-Y_{h}\left(t_{n} ; \mathcal{S}_{h \tau}\left(\Pi_{\tau} U_{h}^{*}\right)\right)\right\|_{\mathbb{L}^{2}}^{2} \mathrm{~d} t\right] \\
& +2 T \max _{0 \leq n \leq N} \mathbb{E}\left[\left\|Y_{h}\left(t_{n} ; \mathcal{S}_{h \tau}\left(\Pi_{\tau} U_{h}^{*}\right)\right)-Y_{h}^{n}\left(\mathcal{S}_{h \tau}\left(\Pi_{\tau} U_{h}^{*}\right)\right)\right\|_{\mathbb{L}^{2}}^{2}\right] .
\end{aligned}
$$


By (4.9), Lemma 3.1 (i), stable property of $(4.15)_{1}$, we can get

$$
\begin{aligned}
& \sum_{n=0}^{N-1} \mathbb{E}\left[\int_{t_{n}}^{t_{n+1}}\left\|Y_{h}\left(t ; \mathcal{S}_{h \tau}\left(\Pi_{\tau} U_{h}^{*}\right)\right)-Y_{h}\left(t_{n} ; \mathcal{S}_{h \tau}\left(\Pi_{\tau} U_{h}^{*}\right)\right)\right\|_{\mathbb{L}^{2}}^{2} \mathrm{~d} t\right] \\
& \quad \leq C \tau\left(\mathbb{E}\left[\left\|\mathcal{S}_{h \tau}\left(\Pi_{\tau} U_{h}^{*}\right)(T)\right\|_{\mathbb{H}_{0}^{1}}^{2}\right]+\|\widetilde{X}(T)\|_{\mathbb{H}_{0}^{1}}^{2}+\left\|\mathcal{S}_{h \tau}\left(\Pi_{\tau} U_{h}^{*}\right)\right\|_{L_{\mathbb{P}}^{2}\left(\Omega ; L^{2}\left(0, T ; \mathbb{L}^{2}\right)\right)}^{2}+\|\widetilde{X}\|_{L^{2}\left(0, T ; \mathbb{L}^{2}\right)}^{2}\right) \\
& \quad \leq C \tau\left(\left\|X_{0}\right\|_{\mathbb{H}_{0}^{1}}^{2}+\|\widetilde{X}(T)\|_{\mathbb{H}_{0}^{1}}^{2}+\|\sigma\|_{L_{\mathbb{F}}^{2}\left(\Omega ; L^{2}\left(0, T ; \mathbb{H}_{0}^{1}\right)\right)}^{2}+\left\|Y_{h}\right\|_{L_{\mathbb{P}}^{2}\left(\Omega ; L^{2}\left(0, T ; \mathbb{H}_{0}^{1}\right)\right)}^{2}+\|\widetilde{X}\|_{L^{2}\left(0, T ; \mathbb{L}^{2}\right)}^{2}\right) \\
& \quad \leq C \tau .
\end{aligned}
$$

Utilizing Theorem 3.5 for $\mathbf{B S P D E}_{h}(2.14)$ with $\Pi_{h} f=\mathcal{S}_{h \tau}\left(\Pi_{\tau} U_{h}^{*}\right)-\Pi_{h}^{1} \widetilde{X}$ and (4.25), we can find that

$$
\begin{aligned}
\max _{0 \leq n \leq N} \mathbb{E}\left[\left\|Y_{h}\left(t_{n} ; \mathcal{S}_{h \tau}\left(\Pi_{\tau} U_{h}^{*}\right)\right)-Y_{h}^{n}\left(\mathcal{S}_{h \tau}\left(\Pi_{\tau} U_{h}^{*}\right)\right)\right\|_{\mathbb{L}^{2}}^{2}\right] \\
\leq C \mathbb{E}\left[\sum_{n=0}^{N-1} \int_{t_{n}}^{t_{n+1}}\left\|\nabla\left[Y_{h}\left(s ; \mathcal{S}_{h \tau}\left(\Pi_{\tau} U_{h}^{*}\right)\right)-Y_{h}\left(t_{n} ; \mathcal{S}_{h \tau}\left(\Pi_{\tau} U_{h}^{*}\right)\right)\right]\right\|_{\mathbb{L}^{2}}^{2}+\left\|\widetilde{X}(s)-\widetilde{X}\left(t_{n}\right)\right\|_{\mathbb{L}^{2}}^{2} \mathrm{~d} s\right] \\
\leq C \tau\left[\max _{0 \leq n \leq N} \mathbb{E}\left[\left\|\nabla X_{h \tau}\left(t_{n} ; \mathcal{S}_{h \tau}\left(\Pi_{\tau} U_{h}^{*}\right)\right)\right\|_{\mathbb{L}^{2}}^{2}\right]+\mathbb{E} \int_{0}^{T}\left\|\nabla \Pi_{h}^{1} \widetilde{X}(t)\right\|_{\mathbb{L}^{2}}^{2} \mathrm{~d} t\right] \\
\quad+C \mathbb{E}\left[\sum_{n=0}^{N-1} \int_{t_{n}}^{t_{n+1}}\left\|\widetilde{X}(s)-\widetilde{X}\left(t_{n}\right)\right\|_{\mathbb{L}^{2}}^{2} \mathrm{~d} s\right] \\
\leq C \tau .
\end{aligned}
$$

Here, we apply the representation of $X_{h \tau}$ (4.18), the fact $\widetilde{X} \in L^{2}\left(0, T ; \mathbb{H}_{0}^{1}\right)$, and condition (4.25).

Now we insert above estimates into (4.28) to obtain assertion (i).

Step 2. For all $k=0,1, \ldots, N$, we define $e_{X}^{k}=X_{h}^{*}\left(t_{k}\right)-X_{h \tau}^{*}\left(t_{k}\right)$. Subtracting (4.14) from (4.5) leads to

$$
\begin{aligned}
e_{X}^{k+1}-e_{X}^{k}= & \tau \Delta_{h} e_{X}^{k+1}+\tau \Pi_{h}^{1}\left[U_{h}^{*}\left(t_{k}\right)-U_{h \tau}^{*}\left(t_{k}\right)\right]+\int_{t_{k}}^{t_{k+1}} \Pi_{h}^{1}\left[\sigma(s)-\sigma\left(t_{k}\right)\right] \mathrm{d} W(s) \\
& +\int_{t_{k}}^{t_{k+1}}\left(\Delta_{h}\left[X_{h}^{*}(s)-X_{h}^{*}\left(t_{k+1}\right)\right]+\Pi_{h}^{1}\left[U_{h}^{*}(s)-U_{h}^{*}\left(t_{k}\right)\right]\right) \mathrm{d} s
\end{aligned}
$$

Testing with $e_{X}^{k+1}$, and using binomial formula, Poincaré's inequality, independence, and absorption lead to

$$
\begin{aligned}
\frac{1}{2} \mathbb{E}[ & \left.\left\|e_{X}^{k+1}\right\|_{\mathbb{L}^{2}}^{2}-\left\|e_{X}^{k}\right\|_{\mathbb{L}^{2}}^{2}+\frac{1}{2}\left\|e_{X}^{k+1}-e_{X}^{k}\right\|_{\mathbb{L}^{2}}^{2}\right]+\frac{\tau}{2} \mathbb{E}\left[\left\|\nabla e_{X}^{k+1}\right\|_{\mathbb{L}^{2}}^{2}\right] \\
\leq & C \tau \mathbb{E}\left[\left\|U_{h}^{*}\left(t_{k}\right)-U_{h \tau}^{*}\left(t_{k}\right)\right\|_{\mathbb{L}^{2}}^{2}\right]+C \mathbb{E}\left[\left\|\int_{t_{k}}^{t_{k+1}} \Pi_{h}^{1}\left[\sigma(s)-\sigma\left(t_{k}\right)\right] \mathrm{d} W(s)\right\|_{\mathbb{L}^{2}}^{2}\right] \\
& +C \mathbb{E}\left[\int_{t_{k}}^{t_{k+1}}\left\|\nabla\left[X_{h}^{*}(s)-X_{h}^{*}\left(t_{k+1}\right)\right]\right\|_{\mathbb{L}^{2}}^{2} \mathrm{~d} s\right]+C \mathbb{E}\left[\int_{t_{k}}^{t_{k+1}}\left\|U_{h}^{*}(s)-U_{h}^{*}\left(t_{k}\right)\right\|_{\mathbb{L}^{2}}^{2} \mathrm{~d} s\right] .
\end{aligned}
$$


By taking the sum over all $0 \leq k \leq n$ and $0 \leq k \leq N-1$, and noting that $e_{X}^{0}=0$, we find that

$$
\begin{aligned}
\max _{0 \leq n \leq N} \mathbb{E}\left[\left\|e_{X}^{n}\right\|_{\mathbb{L}^{2}}^{2}\right]+\sum_{n=1}^{N} \tau \mathbb{E}\left[\left\|\nabla e_{X}^{n}\right\|_{\mathbb{L}^{2}}^{2}\right] \\
\leq C \sum_{k=0}^{N-1} \mathbb{E}\left[\tau\left\|U_{h}^{*}\left(t_{k}\right)-U_{h \tau}^{*}\left(t_{k}\right)\right\|_{\mathbb{L}^{2}}^{2}+\int_{t_{k}}^{t_{k+1}}\left(\left\|\sigma(s)-\sigma\left(t_{k}\right)\right\|_{\mathbb{L}^{2}}^{2}\right.\right. \\
\left.\left.\quad+\left\|\nabla\left[X_{h}^{*}(s)-X_{h}^{*}\left(t_{k+1}\right)\right]\right\|_{\mathbb{L}^{2}}^{2}+\left\|U_{h}^{*}(s)-U_{h}^{*}\left(t_{k}\right)\right\|_{\mathbb{L}^{2}}^{2}\right) \mathrm{~d} s\right] .
\end{aligned}
$$

By (4.28), the first term on the right-hand side is bounded by $C \tau$. We use Itô isometry for the second term, and Hölder regularity in time of $\sigma$ to bound it equally. Adopting the method in (4.31), we can bound the third term by $C \tau\left(\left\|\Delta_{h} X_{h}^{*}(0)\right\|_{\mathbb{L}^{2}}^{2}+\left\|\nabla \Pi_{h}^{1} U_{h}^{*}(t)\right\|_{L_{\mathbb{F}}^{2}\left(\Omega ; L^{2}\left(0, T ; \mathbb{L}^{2}\right)\right)}^{2}+\|\sigma(t)\|_{L_{\mathbb{F}}^{2}\left(\Omega ; L^{2}\left(0, T ; \mathbb{H}^{2}\right)\right)}^{2}\right)$. We use (4.31) to bound the last term by $C \tau$. That is assertion (ii).

Step 3. Firstly, we introduce an auxiliary BSDE

$$
\left\{\begin{aligned}
Y_{\tau}\left(t_{n+1}\right)-Y_{\tau}\left(t_{n}\right)=\tau[ & \left.-\Delta_{h} Y_{\tau}\left(t_{n}\right)+\left[X_{h \tau}^{*}\left(t_{n+1}\right)-\Pi_{h}^{1} \tilde{X}\left(t_{n+1}\right)\right]\right] \\
& +\int_{t_{n}}^{t_{n+1}} Z_{\tau}(t) \mathrm{d} W(t) \quad n=0,1, \ldots, N-1, \\
Y_{\tau}(T)=-\alpha\left(X_{h \tau}^{*}(T)-\right. & \left.\Pi_{h}^{1} \widetilde{X}(T)\right) .
\end{aligned}\right.
$$

It is easy to see that $Y_{\tau}=Y_{h \tau}$. Define $e_{Y}^{n}=Y_{h}\left(t_{n}\right)-Y_{\tau}\left(t_{n}\right), n=0,1, \ldots, N$. With the same argument as that in the proof of Theorem 3.5, we can deduce

$$
\begin{aligned}
& \max _{0 \leq n \leq N} \mathbb{E}\left[\left\|e_{Y}^{n}\right\|_{\mathbb{L}^{2}}^{2}\right]+\sum_{n=1}^{N} \tau \mathbb{E}\left[\left\|\nabla e_{Y}^{n}\right\|_{\mathbb{L}^{2}}^{2}\right] \\
\leq & C \sum_{k=0}^{N-1} \int_{t_{k}}^{t_{k+1}} \mathbb{E}\left[\left\|\nabla\left[Y_{h}(s)-Y_{h}\left(t_{k}\right)\right]\right\|_{\mathbb{L}^{2}}^{2}+\left\|X_{h}^{*}(s)-X_{h \tau}^{*}\left(t_{k+1}\right)\right\|_{\mathbb{L}^{2}}^{2}+\left\|\widetilde{X}(s)-\widetilde{X}\left(t_{k+1}\right)\right\|_{\mathbb{L}^{2}}^{2}\right] \mathrm{d} s .
\end{aligned}
$$

Applying Lemma 3.1 (ii), the first integral term is bounded by

$$
C \tau\left[\left\|\Delta_{h} X_{h}(0)\right\|_{\mathbb{L}^{2}}^{2}+\left\|\Delta_{h} \Pi_{h}^{1} \widetilde{X}(T)\right\|_{\mathbb{L}^{2}}^{2}+\int_{0}^{T} \mathbb{E}\left[\left\|\nabla X_{h}^{*}(t)\right\|_{\mathbb{L}^{2}}^{2}+\left\|\nabla \Pi_{h}^{1} \widetilde{X}_{h}^{*}(t)\right\|_{\mathbb{L}^{2}}^{2}+\left\|\Pi_{h}^{1} U_{h}^{*}(t)\right\|_{\mathbb{L}^{2}}^{2}\right] \mathrm{d} t\right] .
$$

It remains to estimate the second integral term, which is bounded by

$$
\begin{aligned}
C & \sum_{k=0}^{N-1} \int_{t_{k}}^{t_{k+1}} \mathbb{E}\left[\left\|X_{h}^{*}(s)-X_{h}^{*}\left(t_{k+1}\right)\right\|_{\mathbb{L}^{2}}^{2}+\left\|X_{h}^{*}\left(t_{k+1}\right)-X_{h \tau}^{*}\left(t_{k+1}\right)\right\|_{\mathbb{L}^{2}}^{2}\right] \mathrm{d} s \\
\leq & C \tau\left[\left\|\nabla X_{h}(0)\right\|_{\mathbb{L}^{2}}^{2}+\int_{0}^{T} \mathbb{E}\left[\left\|\Pi_{h}^{1} U_{h}^{*}(t)\right\|_{\mathbb{L}^{2}}^{2}+\left\|\nabla \Pi_{h}^{1} \sigma(t)\right\|_{\mathbb{L}^{2}}^{2}\right] \mathrm{d} t\right] \\
& \quad+C \max _{0 \leq k \leq N} \mathbb{E}\left[\left\|X_{h}^{*}\left(t_{k}\right)-X_{h \tau}^{*}\left(t_{k}\right)\right\|_{\mathbb{L}^{2}}^{2}\right] \\
\leq & C \tau .
\end{aligned}
$$

Assertion (iii) now follows from the above three statements and conditions on $X_{0}, \sigma, \widetilde{X}$. 
Remark 4.4. In this work, we apply implicit Euler method to solve $\mathbf{B S P D E}_{h}$ (2.14) and (4.7). In the literature, some higher order schemes exist for the simulation of BSDEs of fixed, finite dimension; see, e.g., [9, 37, 41]. These schemes are also candidates to solve problem SLQ, and this is a meaningful research topic.

Remark 4.5. Error analysis in this section can be trivially transferred to some generalized SLQ problems. For example, the cost functional and controlled equation could be

$$
\begin{aligned}
\mathcal{J}(X, U)= & \frac{1}{2} \mathbb{E}\left[\int_{0}^{T} \int_{D}\left(\alpha_{X}(t, x)|X(t, x)-\tilde{X}(t, x)|^{2}+\alpha_{U}(t, x)|U(t, x)|^{2}\right) \mathrm{d} x \mathrm{~d} t\right. \\
& \left.+\int_{D} \alpha(x)|X(T, x)-\tilde{X}(T, x)|^{2} \mathrm{~d} x\right]
\end{aligned}
$$

and

$$
\begin{cases}\mathrm{d} X(t, x)=[-\mathcal{L} X(t, x)+U(t, x)] \mathrm{d} t+\sigma(t, x) \mathrm{d} W(t) & (t, x) \in[0, T] \times D \\ X(t, x)=0 & (t, x) \in(0, T) \times \partial D \\ X(0, x)=X_{0}(x) & x \in D\end{cases}
$$

where $\mathcal{L} X=-\sum_{i, j=1}^{d} \frac{\partial}{\partial x_{i}}\left(a_{i j}(x) \frac{\partial X}{\partial x_{j}}\right)+c(x) X,\left(a_{i j}\right)$ is symmetric and uniformly elliptic, $\alpha_{X}, \alpha_{U}, \alpha, c$ are positive bounded functions, and $\alpha_{U} \geq \delta>0$. Besides, based on Pontryagin's maximum principle, the spatiotemporal discretization strategy for problem SLQ can also be utilized to general stochastic optimal control problems with stochastic parabolic equations constraints.

\section{The GRAdient Descent Method to SOLve $\mathbf{S L Q}_{h \tau}$}

By Theorem 4.2, solving minimization problem $\mathbf{S L Q} \mathbf{Q}_{h \tau}$ is equivalent to solving the system of coupled forwardbackward difference equations (4.15) and (4.16). We may exploit the variational character of problem SLQ $\mathbf{Q}_{h \tau}$ to construct a gradient descent method $\mathbf{S L Q} \mathbf{Q}_{h \tau}^{\text {grad }}$ where approximate iterates of the optimal control $U_{h \tau}^{*}$ in the Hilbert space $\mathbb{U}_{h \tau}$ are obtained; see also $[23,30]$.

Algorithm 5.1. $\left(\mathbf{S L Q}_{h \tau}^{\mathrm{grad}}\right)$ Let $U_{h \tau}^{(0)} \in \mathbb{U}_{h \tau}$, and fix $\kappa>0$. For any $\ell \in \mathbb{N}_{0}$, update $U_{h \tau}^{(\ell)} \in \mathbb{U}_{h \tau}$ as follows:

1. Compute $X_{h \tau}^{(\ell)} \in \mathbb{X}_{h \tau}$ by

$$
\left\{\begin{array}{l}
{\left[\mathbb{1}-\tau \Delta_{h}\right] X_{h \tau}^{(\ell)}\left(t_{n+1}\right)=X_{h \tau}^{(\ell)}\left(t_{n}\right)+\tau \Pi_{h}^{1} U_{h \tau}^{(\ell)}\left(t_{n}\right)+\Pi_{h}^{1} \sigma\left(t_{n}\right) \Delta_{n+1} W \quad n=0,1, \ldots, N-1,} \\
X_{h \tau}^{(\ell)}(0)=\Pi_{h}^{1} X_{0} .
\end{array}\right.
$$

2. Use $X_{h \tau}^{(\ell)} \in \mathbb{X}_{h \tau}$ to compute $Y_{h \tau}^{(\ell)} \in \mathbb{X}_{h \tau}$ via

$$
\left\{\begin{array}{c}
{\left[\mathbb{1}-\tau \Delta_{h}\right] Y_{h \tau}^{(\ell)}\left(t_{n}\right)=\mathbb{E}\left[Y_{h \tau}^{(\ell)}\left(t_{n+1}\right)-\tau\left(X_{h \tau}^{(\ell)}\left(t_{n+1}\right)-\Pi_{h}^{1} \tilde{X}\left(t_{n+1}\right)\right) \mid \mathcal{F}_{t_{n}}\right]} \\
n=0,1, \ldots, N-1, \\
Y_{h \tau}^{(\ell)}(T)=-\alpha\left(X_{h \tau}^{(\ell)}(T)-\Pi_{h}^{1} \tilde{X}(T)\right) .
\end{array}\right.
$$

3. Compute the update $U_{h \tau}^{(\ell+1)} \in \mathbb{U}_{h \tau}$ via

$$
U_{h \tau}^{(\ell+1)}=U_{h \tau}^{(\ell)}-\frac{1}{\kappa}\left(U_{h \tau}^{(\ell)}-\Pi_{h}^{0} Y_{h \tau}^{(\ell)}\right) .
$$


Note that Steps 1 and 2 are now decoupled: the first step requires to solve a space-time discretization (2.7) of SPDE (2.3), while the second requires to solve the space-time discretization $(3.6)_{1}$ of the BSPDE (4.1) $)_{2}$. We refer to related works on how to approximate conditional expectations [3, 6, 18, 24, 33]; a similar method to $\mathbf{S L Q}_{h \tau}^{\text {grad }}$ to solve problem $\mathbf{S L Q} \mathbf{Q}_{h \tau}$ has been proposed in [15]. For general optimal control problem, to reduce computation cost, one can adopt stochastic gradient descent methods; see e.g. [1].

Remark 5.2. In [24], based on variational method, a finite transposition method for solving BSPDEs like $(4.1)_{2}$ is proposed, which avoids the computation of conditional expectations via Monte-Carlo simulation; see [24] for further details.

We want to show convergence of $\mathbf{S L Q}_{h \tau}^{\text {grad }}$ for $\kappa>0$ sufficiently large and $\ell \uparrow \infty$. For this purpose, we recall the notations $\mathcal{S}_{h \tau}, \mathcal{T}_{h \tau}, \widehat{\mathcal{J}}_{h \tau}$ introduced in Section 4.2. For this purpose, we first recall Lipschitz continuity of $D \widehat{\mathcal{J}}_{h \tau}:$ since

$$
D^{2} \widehat{\mathcal{J}}_{h \tau}\left(U_{h \tau}\right)=\left(\mathbb{1}+L^{*} L+\alpha \widehat{L}^{*} \widehat{L}\right) U_{h \tau},
$$

where operators $L, \widehat{L}$ are defined in (4.18), we find $K:=\left\|\mathbb{1}+L^{*} L+\alpha \widehat{L} * \widehat{L}\right\|_{\mathcal{L}\left(\mathbb{U}_{h \tau} ; \mathbb{U}_{h \tau}\right)}$, such that

$$
\left\|D \widehat{\mathcal{J}}_{h \tau}\left(U_{h \tau}^{1}\right)-D \widehat{\mathcal{J}}_{h \tau}\left(U_{h \tau}^{2}\right)\right\|_{\mathbb{U}_{h \tau}} \leq K\left\|U_{h \tau}^{1}-U_{h \tau}^{2}\right\|_{\mathbb{U}_{h \tau}} .
$$

Indeed, noting that $\left\|\left(\mathbb{1}-\tau \Delta_{h}\right)^{-1}\right\|_{\mathcal{L}\left(\mathbb{V}_{h}^{1} ; \mathbb{V}_{h}^{1}\right)} \leq 1$, we conclude

$$
\left\|L U_{h \tau}\right\|_{\mathbb{X}_{h \tau}}^{2}=\sum_{n=1}^{N} \tau \mathbb{E}\left[\left\|L U_{h \tau}\left(t_{n}\right)\right\|_{\mathbb{L}^{2}}^{2}\right]=\sum_{n=1}^{N} \tau \mathbb{E}\left[\left\|\tau \sum_{j=0}^{n-1}\left[\left(\mathbb{1}-\tau \Delta_{h}\right)^{-1}\right]^{n-j} \Pi_{h}^{1} U_{h \tau}\left(t_{j}\right)\right\|_{\mathbb{L}^{2}}^{2}\right] \leq T^{2}\left\|U_{h \tau}\right\|_{\mathbb{U}_{h \tau}}^{2},
$$

and

$$
\left\|\widehat{L} U_{h \tau}\right\|_{L_{\mathcal{F}_{T}}^{2}\left(\Omega ; \mathbb{L}^{2}\right)}^{2}=\mathbb{E}\left[\left\|\tau \sum_{j=0}^{N-1}\left[\left(\mathbb{1}-\tau \Delta_{h}\right)^{-1}\right]^{N-j} \Pi_{h}^{1} U_{h \tau}\left(t_{j}\right)\right\|_{\mathbb{L}^{2}}^{2}\right] \leq T\left\|U_{h \tau}\right\|_{\mathbb{U}_{h \tau}}^{2} .
$$

Hence

$$
K=\left\|\mathbb{1}+L^{*} L+\alpha \widehat{L}^{*} \widehat{L}\right\|_{\mathcal{L}\left(\mathbb{U}_{h \tau} ; \mathbb{U}_{h \tau}\right)} \leq 1+\alpha T+T^{2} .
$$

Since $\mathbf{S L Q} \mathbf{Q}_{h \tau}^{\text {grad }}$ is the gradient descent method for $\mathbf{S L} \mathbf{Q}_{h \tau}$, we have the following result.

Theorem 5.3. Suppose that $\kappa \geq K$. Let $\left\{U_{h \tau}^{(\ell)}\right\}_{\ell \in \mathbb{N}_{0}} \subset \mathbb{U}_{h \tau}$ be generated by $\mathbf{S L Q}_{h \tau}^{\mathrm{grad}}$, and $U_{h \tau}^{*}$ solve $\mathbf{S L Q} \mathbf{Q}_{h \tau}$. Then

$$
\left\{\begin{array}{l}
\widehat{\mathcal{J}}_{h \tau}\left(U_{h \tau}^{(\ell)}\right)-\widehat{\mathcal{J}}_{h \tau}\left(U_{h \tau}^{*}\right) \leq \frac{2 \kappa\left\|U_{h \tau}^{(0)}-U_{h \tau}^{*}\right\|_{\mathbb{U}_{h \tau}}^{2}}{\ell}, \\
\left\|U_{h \tau}^{(\ell)}-U_{h \tau}^{*}\right\|_{\mathbb{U}_{h \tau}}^{2} \leq\left(1-\frac{1}{\kappa}\right)^{\ell}\left\|U_{h \tau}^{(0)}-U_{h \tau}^{*}\right\|_{\mathbb{U}_{h \tau}}^{2} \quad \ell=1,2, \ldots
\end{array}\right.
$$

Proof. We know that $D \widehat{\mathcal{J}}_{h \tau}$ is Lipschitz continuous with constant $K>0$. Also, $\widehat{\mathcal{J}}_{h \tau}$ is strongly convex. Hence, the gradient descent method in abstract form is the following iteration (see Algorithm 5.1, Step 3.)

$$
U_{h \tau}^{(\ell+1)}=U_{h \tau}^{(\ell)}-\frac{1}{\kappa} D \widehat{\mathcal{J}}_{h \tau}\left(U_{h \tau}^{(\ell)}\right), \quad \ell=0,1,2 \cdots .
$$


By the proof of Theorem 4.2, we have obtained the following facts:

$$
\left\{\begin{array}{l}
D \widehat{\mathcal{J}}_{h \tau}\left(U_{h \tau}^{(\ell)}\right)=U_{h \tau}^{(\ell)}-\Pi_{h}^{0} \mathcal{T}_{h \tau}\left(\mathcal{S}_{h \tau}\left(U_{h \tau}^{(\ell)}\right)\right), \\
\Pi_{h}^{0} \mathcal{T}_{h \tau}\left(\mathcal{S}_{h \tau}\left(U_{h \tau}^{(\ell)}\right)\right)=-L^{*}\left(\Gamma \Pi_{h}^{1} X_{0}+L U_{h \tau}^{(\ell)}+f-\widetilde{X}\right)-\alpha \widehat{L}^{*}\left(\widehat{\Gamma} \Pi_{h}^{1} X_{0}+\widehat{L} U_{h \tau}^{(\ell)}+\widehat{f}-\widetilde{X}(T)\right),
\end{array}\right.
$$

where $L, \widehat{L}, \Gamma, \widehat{\Gamma}, \widehat{f}$ are defined in (4.18) and (4.19). Via (4.20), we have that $\Pi_{h}^{0} \mathcal{T}_{h \tau}\left(\mathcal{S}_{h \tau}\left(U_{h \tau}^{(\ell)}\right)\right)$ is just $Y_{h \tau}^{(\ell)}$, the solution of Step 2 in Algorithm 5.1. Therefore, (5.1) is consistent with the gradient descent method $\mathbf{S L Q}_{h \tau}^{\mathrm{grad}}$. The desired error estimates now follow by standard estimates for the gradient descent method (see e.g. [30], Thm. 1.2.4).

Acknowledgements. This work was carried out when Yanqing Wang visited the University of Tübingen in 2019-2020, and he gratefully acknowledges the support of K. C. Wong Education Foundation and DAAD.

\section{REFERENCES}

[1] R. Archibald, F. Bao and J. Yong, A stochastic gradient descent approach for stochastic optimal control. East Asian J. Appl. Math. 10 (2020) 635-658.

[2] R. Archibald, F. Bao, J. Yong and T. Zhou, An efficient numerical algorithm for solving data driven feedback control problems J. Sci. Comput. 85 (2020) Paper No. 51, 27.

[3] C. Bender and R. Denk, A forward scheme for backward SDEs. Stochastic Process. Appl. 117 (2007) $1793-1812$.

[4] C. Bender and J. Zhang, Time discretization and Markovian iteration for coupled FBSDEs. Ann. Appl. Probab. 18 (2008) $143-177$.

[5] A. Bensoussan, Stochastic maximum principle for distributed parameter systems. J. Franklin Inst. 315 (1983) $387-406$.

[6] B. Bouchard and N. Touzi, Discrete-time approximation and Monte-Carlo simulation of backward stochastic differential equations. Stochastic Process. Appl. 111 (2004) 175-206.

[7] J.H. Bramble, J.E. Pasciak and O. Steinbach, On the stability of the $L^{2}$ projection in $H^{1}(\Omega)$. Math. Comp. 71 (2002) $147-156$.

[8] S.C. Brenner and L.R. Scott, The mathematical theory of finite element methods. Vol. 15 of Texts in Applied Mathematics. Springer, New York, third ed. (2008).

[9] J.-F. Chassagneux, Linear multistep schemes for BSDEs. SIAM J. Numer. Anal. 52 (2014) 2815-2836.

[10] M. Crouzeix and V. Thomée, The stability in $L_{p}$ and $W_{p}^{1}$ of the $L_{2}$-projection onto finite element function spaces. Math. Comp. 48 (1987) 521-532.

[11] L. Dai, Y. Zhang and J. Zou, Numerical schemes for forward-backward stochastic differential equations using transposition solutions (2017) preprint.

[12] F. Dou and Q. Lü, Partial approximate controllability for linear stochastic control systems. SIAM J. Control Optim. 57 (2019) $1209-1229$.

[13] K. Du, $W^{2, p}$-solutions of parabolic SPDEs in general domains. Stochastic Process. Appl. 130 (2020) 1-19.

[14] K. Du and S. Tang, Strong solution of backward stochastic partial differential equations in $C^{2}$ domains. Probab. Theory Related Fields 154 (2012) 255-285.

[15] T. Dunst and A. Prohl, The forward-backward stochastic heat equation: numerical analysis and simulation. SIAM J. Sci. Comput. 38 (2016) A2725-A2755.

[16] W. E, M. Hutzenthaler, A. Jentzen and T. Kruse, On multilevel Picard numerical approximations for high-dimensional nonlinear parabolic partial differential equations and high-dimensional nonlinear backward stochastic differential equations. J. Sci. Comput. 79 (2019) 1534-1571.

[17] N. El Karoui, S. Peng and M.C. Quenez, Backward stochastic differential equations in finance. Math. Finance 7 (1997) 1-71.

[18] E. Gobet, J.-P. Lemor and X. Warin, A regression-based Monte Carlo method to solve backward stochastic differential equations. Ann. Appl. Probab. 15 (2005) 2172-2202.

[19] B. Gong, W. Liu, T. Tang, W. Zhao and T. Zhou, An efficient gradient projection method for stochastic optimal control problems. SIAM J. Numer. Anal. 55 (2017) 2982-3005.

[20] W. Gong and M. Hinze, Error estimates for parabolic optimal control problems with control and state constraints. Comput. Optim. Appl. 56 (2013) 131-151.

[21] M. Hinze, R. Pinnau, M. Ulbrich and S. Ulbrich, Optimization with PDE constraints. Vol. 23 of Mathematical Modelling: Theory and Applications. Springer, New York (2009).

[22] Y. Hu, D. Nualart and X. Song, Malliavin calculus for backward stochastic differential equations and application to numerical solutions. Ann. Appl. Probab. 21 (2011) 2379-2423.

[23] S.I. Kabanikhin, Inverse and ill-posed problems. Vol. 55 of Inverse and Ill-posed Problems Series. Walter de Gruyter GmbH \& Co. KG, Berlin (2012).

[24] Q. Lü, P. Wang, Y. Wang and X. Zhang, Numerics for stochastic distributed parameter control systems: a finite transposition method. arXiv:2104.02964 (2020). 
[25] Q. Lü and X. Zhang, General Pontryagin-type stochastic maximum principle and backward stochastic evolution equations in infinite dimensions. SpringerBriefs in Mathematics, Springer, Cham (2014).

[26] Q. Lü and X. Zhang, Mathematical control theory for stochastic partial differential equations. Springer (in press).

[27] K. Malanowski, Convergence of approximations vs. regularity of solutions for convex, control-constrained optimal-control problems. Appl. Math. Optim. 8 (1982) 69-95.

[28] R.S. McKnight and W.E. Bosarge, Jr., The Ritz-Galerkin procedure for parabolic control problems. SIAM J. Control 11 (1973) 510-524.

[29] D. Meidner and B. Vexler, A priori error estimates for space-time finite element discretization of parabolic optimal control problems. I. Problems without control constraints. SIAM J. Control Optim. 47 (2008) 1150-1177.

[30] Y. Nesterov, Introductory lectures on convex optimization. Vol. 87 of Applied Optimization. Kluwer Academic Publishers, Boston, MA (2004).

[31] D. Nualart, The Malliavin calculus and related topics. Probability and its Applications (New York), Springer-Verlag, Berlin, second ed. (2006).

[32] A. Rösch, Error estimates for parabolic optimal control problems with control constraints. Z. Anal. Anwendungen 23 (2004) 353-376.

[33] P. Wang and X. Zhang, Numerical solutions of backward stochastic differential equations: a finite transposition method. $C$. R. Math. Acad. Sci. Paris 349 (2011) 901-903.

[34] Y. Wang, Transposition solutions of backward stochastic differential equations and numerical schemes, Ph.D. Thesis, Academy of Mathematics and Systems Science, Chinese Academy of Sciences (2013).

[35] Y. Wang, A semidiscrete Galerkin scheme for backward stochastic parabolic differential equations. Math. Control Relat. Fields 6 (2016) 489-515.

[36] Y. Yan, Galerkin finite element methods for stochastic parabolic partial differential equations. SIAM J. Numer. Anal. 43 (2005) 1363-1384.

[37] J. Yang, W. Zhao and T. Zhou, A unified probabilistic discretization scheme for FBSDEs: stability, consistency, and convergence analysis. SIAM J. Numer. Anal. 58 (2020) 2351-2375.

[38] J. Yong and X.Y. Zhou, Stochastic controls: Hamiltonian systems and HJB equations. Vol. 43 of Applications of Mathematics (New York). Springer-Verlag, New York (1999).

[39] J. Zhang, A numerical scheme for BSDEs. Ann. Appl. Probab. 14 (2004) 459-488.

[40] X. Zhang, Regularities for semilinear stochastic partial differential equations. J. Funct. Anal. 249 (2007) $454-476$.

[41] W. Zhao, L. Chen and S. Peng, A new kind of accurate numerical method for backward stochastic differential equations. SIAM J. Sci. Comput. 28 (2006) 1563-1581. 